\title{
Transition stages of Rayleigh-Taylor instability between miscible fluids
}

\author{
By ANDREW W. COOK ${ }^{1}$ AND PAUL E. DIMOTAKIS \\ ${ }^{1}$ Lawrence Livermore National Laboratory, Livermore, CA 94550, USA \\ ${ }^{2}$ Graduate Aeronautical Laboratories, California Institute of Technology, \\ Pasadena, CA 91125, USA
}

(Received 2 June and in revised form 28 March 2001)

Direct numerical simulations (DNS) are presented of three-dimensional, RayleighTaylor instability (RTI) between two incompressible, miscible fluids, with a 3:1 density ratio. Periodic boundary conditions are imposed in the horizontal directions of a rectangular domain, with no-slip top and bottom walls. Solutions are obtained for the Navier-Stokes equations, augmented by a species transport-diffusion equation, with various initial perturbations. The DNS achieved outer-scale Reynolds numbers, based on mixing-zone height and its rate of growth, in excess of 3000. Initial growth is diffusive and independent of the initial perturbations. The onset of nonlinear growth is not predicted by available linear-stability theory. Following the diffusive-growth stage, growth rates are found to depend on the initial perturbations, up to the end of the simulations. Mixing is found to be even more sensitive to initial conditions than growth rates. Taylor microscales and Reynolds numbers are anisotropic throughout the simulations. Improved collapse of many statistics is achieved if the height of the mixing zone, rather than time, is used as the scaling or progress variable. Mixing has dynamical consequences for this flow, since it is driven by the action of the imposed acceleration field on local density differences.

\section{Introduction}

Rayleigh-Taylor instability (RTI) occurs whenever fluids of different density are subjected to acceleration in a direction opposite that of the density gradient (Rayleigh 1883; Taylor 1950; Chandrasekhar 1955, 1961; Duff, Harlow \& Hurt 1962; Sharp 1984). RTI is encountered in a variety of contexts, such as combustion, rotating machinery, inertial-confinement fusion, supernovae explosions, and geophysics. The Richtmyer-Meshkov instability (RMI, Richtmyer 1960; Meshkov 1969) is closely related and may be regarded as a special case of RTI; it corresponds to the case of an impulsive acceleration. Both flows can become turbulent, as secondary, KelvinHelmholtz instabilities (KHI) develop that broaden the spectrum of spatial and temporal scales participating in the dynamics.

RTI flows represent an important simulation and modelling test case for hydrodynamic codes, in general, involving many aspects of turbulence, transport, and diffusion in non-uniform-density flows subject to external body forces. RTI is driven by a directed forcing term, scaled by local density differences. This flow is thus capable of sustaining anisotropy in fully developed turbulence. Secondly, for miscible fluids, species diffusion, which reduces density differences and hence local forcing, plays a dynamic role in this flow and must be captured to simulate the full dynamics. 
One class of models for RTI in the turbulent regime assumes that the mixing zone grows quadratically in time (Annuchina et al. 1978; Read 1984; Youngs 1984, 1989). For a constant and uniform acceleration, $g$, the vertical penetration, $h_{\mathrm{b}}(t)$, of the light fluid into the heavy fluid (dubbed 'bubbles'), is modelled as

$$
h_{\mathrm{b}}(t) \simeq \alpha_{\mathrm{b}} \mathscr{A} g t^{2} .
$$

The penetration, $h_{\mathrm{s}}(t)$, of the advancing heavy into light fluid ('spikes'), is similarly modelled

$$
h_{\mathrm{s}}(t) \simeq-\alpha_{\mathrm{s}} \mathscr{A} g t^{2}
$$

where the subscripts $\mathrm{b}$ and $\mathrm{s}$ denote bubbles and spikes, and $\alpha_{\mathrm{b}}$ and $\alpha_{\mathrm{s}}$ are model constants. The parameter $\mathscr{A}$ in (1) is the Atwood number, defined as

$$
\mathscr{A} \equiv \frac{\rho_{2}-\rho_{1}}{\rho_{2}+\rho_{1}}=\frac{R-1}{R+1}, \quad R=\frac{\rho_{2}}{\rho_{1}},
$$

with $\rho_{2}$ and $\rho_{1}$ the densities of the heavy and light fluids, respectively. The total extent, $h(t)$, of the RTI mixing zone can be defined as

$$
h(t)=h_{\mathrm{b}}(t)-h_{\mathrm{s}}(t)
$$

and, from (1),

$$
h(t) \simeq \alpha \mathscr{A} g t^{2},
$$

where $\alpha=\alpha_{\mathrm{b}}+\alpha_{\mathrm{s}}$. The rate of growth of the mixing zone can then be estimated by

$$
\dot{h}=\frac{\mathrm{d} h}{\mathrm{~d} t}=2 \alpha \mathscr{A} g t=2(\alpha \mathscr{A} g h)^{1 / 2} .
$$

As discussed by Dimonte \& Schneider (2000), experimental and computational estimates for $\alpha$ span a fairly large range, $0.01 \lesssim \alpha \lesssim 0.07$.

A heuristic model for the growth of the mixing zone can be outlined as follows. The advancement of RTI bubbles and spikes in the respective pure fluids may be viewed as the result of a buoyancy force scaled by the density difference across the corresponding front, i.e.

$$
\bar{\rho} \frac{\mathrm{d} u_{i}}{\mathrm{~d} t}=\bar{\rho} \frac{\mathrm{d} \dot{h}_{i}}{\mathrm{~d} t} \simeq \pm \alpha_{i} \Delta \rho g,
$$

where $\bar{\rho}=\left(\rho_{2}+\rho_{1}\right) / 2$ and $\Delta \rho=\rho_{2}-\rho_{1}$, for $i=\mathrm{b}, \mathrm{s}$, corresponding to bubbles and spikes, respectively, with the $\alpha_{i}$ representing suitable model constants. Integrating once yields

$$
\dot{h}_{i} \simeq \pm \alpha_{i} \frac{\Delta \rho}{\bar{\rho}} g\left(t+t_{i 0}\right),
$$

and again,

$$
h_{i} \simeq \pm \alpha_{i} \mathscr{A} g\left(t+t_{i 0}\right)^{2}+h_{i 0},
$$

with $\mathscr{A}=\Delta \rho /(2 \bar{\rho})$, as in (2), and $t_{i 0}$ and $h_{i 0}$ integration constants. This recovers $(3 b)$, via $(3 a)$, within the undetermined integration constants. The validity of such a scaling argument hinges on a few implicit assumptions, e.g. that the density difference across the advancing fronts be constant and that the flow structure in the vicinity of the fronts, as scaled by the mixing-zone extent, $h$, be self-similar.

Growth models with additional flexibility have been proposed to accommodate the initial perturbation amplitude as well as pressure and other drag components on the 
advancing structures. In these, the respective front velocities are modelled as

$$
\frac{\mathrm{d} \dot{h}_{i}}{\mathrm{~d} t} \simeq \beta_{i} \mathscr{A} g-C_{i} \frac{\dot{h}_{i}\left|\dot{h}_{i}\right|}{h_{i}} .
$$

The model coefficients, $\beta_{i}$ and $C_{i}$, in this expression are reported to depend on the density ratio, $R$, and $h_{\mathrm{b}} / h_{\mathrm{s}}$. If $\beta_{i}$ and $C_{i}$ are approximated as constants, the previous model parameters are given by

$$
\alpha_{i}=\frac{\beta_{i}}{2\left(1+2 C_{i}\right)},
$$

and $(5 a, b)$ yields the approximate solution

$$
h_{i}(t) \simeq \alpha_{i} \mathscr{A} g\left\{t+\left[1-\psi_{i}(t)\right] t_{i 0}\right\}^{2},
$$

where $t_{i 0}=\sqrt{h_{i 0} /\left(\alpha_{i} \mathscr{A} g\right)}$ and $\psi_{i 0} \ll t / t_{i 0}+1$ (Dimonte \& Schneider 2000), i.e. the same basic result as the scaling argument outlined in (4).

Experimental evidence and numerical simulations of this phenomenon to date have not provided unambiguous support for these model expressions, with variations reportedly dependent on Atwood number (e.g. Dalziel, Linden \& Youngs 1999), initial perturbations (Linden, Redondo \& Youngs 1994), geometry (Andrews \& Spalding 1990), and profile of the density interface (Young et al. 2001). Even less is known about the behaviour for the evolution with variable acceleration, $g=g(t)$, as well as the behaviour in fully developed turbulent flow.

Generally, the growth of the RTI mixing zone between viscous and miscible fluids with light and heavy fluid viscosities $\mu_{1}, \mu_{2}$, respectively, and a (binary) diffusivity $\mathscr{D}$, in a domain of transverse extent, $L$, subject to initial perturbations of a characteristic (dominant-mode) wavelength, $\lambda_{\mathrm{c}}$, can be parameterized as,

$$
h(t)=\mathrm{fn}\left(L, g, t, \lambda_{\mathrm{c}}, \rho_{1}, \rho_{2}, \mu_{1}, \mu_{2}, \mathscr{D}\right),
$$

or, relating the seven possible (but non-unique) dimensionless groups,

$$
h(t) / L=f\left[t / \tau, L / \lambda_{\mathrm{c}}, R, \mu_{2} / \mu_{1}, R e_{h}, S c\right]
$$

with $R$ as in (2),

a characteristic time,

$$
\tau=\left(\frac{L}{\mathscr{A} g}\right)^{1 / 2}
$$

$$
R e_{h}=\frac{\bar{\rho} \dot{h} h}{\bar{\mu}}
$$

the outer-scale Reynolds number of the evolving flow, with $\bar{\rho}$ as in $(4 a)$ and $\bar{\mu}=$ $\left(\mu_{1}+\mu_{2}\right) / 2$, and

$$
S c=\frac{\bar{\mu}}{\bar{\rho} \mathscr{D}}=\frac{v}{\mathscr{D}}
$$

the Schmidt number.

In the limit of $R e_{h} \gg 1$ and $S c \approx 1$, it may be possible to neglect viscous and diffusive effects, removing $R e_{h}$ and $S c$ as important parameters. If the parameterization in the density ratio is captured by the characteristic time scale, $\tau(6 c)$, in which case $R$ would not enter as an independent dimensionless parameter, then we must have $h / L \simeq f(t / \tau)$. Quadratic growth may then be expected if, in addition, $L / \lambda_{\mathrm{c}} \gg 1$, or if $h / L \ll 1$, in which case the transverse extent, $L$, will not be felt. This leads to 
$h / L \propto(t / \tau)^{2}$ as the only possibility that removes $L$ from the dynamics, and $(3 b)$ is recovered.

This paper addresses some of the scaling issues, to the extent feasible by direct numerical simulation (DNS), focusing on the effect of initial conditions on the growth and development of the Rayleigh-Taylor mixing zone. The effects are studied by comparing the response to different perturbations of the initial diffusion interface, each characterized by a different dominant wavenumber, $k_{c}$. In particular, the perturbation spectra are chosen to have dominant wavenumbers smaller than, comparable to, and higher than the most unstable wavenumbers, according to the Duff et al. (1962) linear stability theory.

\section{Problem description}

We report on a study of incompressible RTI flow, with a density ratio, $R=\rho_{2} / \rho_{1}=$ 3 , in a rectangular parallelepiped with square cross-section, subject to an acceleration field in the negative- $z$ direction, i.e.

$$
\boldsymbol{g}=-\hat{z} g .
$$

The flow is investigated through DNS by solving the Navier-Stokes and species transport-diffusion equations.

Diffusion between the two miscible fluids is modelled as Fickian, i.e. with a diffusive mass flux of the heavy fluid given by

$$
\boldsymbol{j}(\boldsymbol{x}, t)=-\rho \mathscr{D} \nabla Y(\boldsymbol{x}, t)=-\frac{\rho_{1} \rho_{2}}{\rho} \mathscr{D} \nabla X(\boldsymbol{x}, t),
$$

where $\rho=\rho(\boldsymbol{x}, t)$ is the fluid density, $\mathscr{D}$ is the binary species diffusivity $(6 a)$, and $Y(\boldsymbol{x}, t)$ and $X(\boldsymbol{x}, t)$ are the heavy-fluid mass fraction and mole fraction, respectively. These are related to density by

$$
\begin{gathered}
X(\boldsymbol{x}, t)=\frac{\rho(\boldsymbol{x}, t)-\rho_{1}}{\rho_{2}-\rho_{1}}, \\
\frac{1}{\rho(\boldsymbol{x}, t)}=\frac{Y(\boldsymbol{x}, t)}{\rho_{2}}+\frac{1-Y(\boldsymbol{x}, t)}{\rho_{1}},
\end{gathered}
$$

with $\rho Y=\rho_{2} X$. Incompressible (uniform number density) fluids are assumed with density fluctuations arising solely from composition variations.

In the simulations presented here, the flow is followed well past the linear-growth regime. Simulation and flow parameters are selected such that spectral resolution limitations towards the end of the simulations are encountered before the RTI mixing zone is affected by the upper or lower walls.

The range of length scales can be characterized by the outer-scale Reynolds number, $R e_{h}(6 d)$. In simulations of viscous/diffusive flow, $R e_{h}$ must be high enough for viscous effects to be relatively unimportant.

\subsection{Governing equations}

Choosing the light-fluid density, $\rho_{1}$, the length $\ell=L /(2 \pi)$, and the acceleration magnitude, $g$, as the scaling parameters, the dimensionless equations governing the flow may be written as (cf. Sandoval 1995),

$$
\frac{\partial \rho}{\partial t}+\frac{\partial \rho u_{j}}{\partial x_{j}}=0
$$


where $\rho$ is the fluid-mixture density, scaled by $\rho_{1} ; t$ is time, scaled by $\sqrt{\ell / g} ; \boldsymbol{u}=$ $\left(u_{1}, u_{2}, u_{3}\right)=(u, v, w)$ is the velocity vector, scaled by $U=\sqrt{g \ell} ; \boldsymbol{x}=\left(x_{1}, x_{2}, x_{3}\right)=$ $(x, y, z)$ is the Cartesian coordinate vector, scaled by $\ell$;

$$
\frac{\partial \rho u_{i}}{\partial t}+\frac{\partial \rho u_{i} u_{j}}{\partial x_{j}}=-\frac{\partial p}{\partial x_{i}}+\frac{\partial \tau_{i j}}{\partial x_{j}}-\rho \delta_{i 3},
$$

where $p$ is the pressure, scaled by $\rho_{1} U^{2}$, with

$$
\tau_{i j}=\frac{1}{R e}\left[\frac{\partial u_{i}}{\partial x_{j}}+\frac{\partial u_{j}}{\partial x_{i}}-\frac{2}{3}(\nabla \cdot u) \delta_{i j}\right]
$$

the scaled stress tensor, where $R e=\rho_{1} \ell U / \mu$ is the scaling Reynolds number, with $\mu$ a viscosity discussed below. In this non-dimensionalization, the Froude number, $F r=U / \sqrt{g \ell}$, whose square typically divides the last term in (11a), is identically equal to unity and does not enter as a parameter.

The species-transport equation, with Fickian fluxes (8) and no interfacial surface tension, is given by

$$
\frac{\partial \rho Y}{\partial t}+\frac{\partial \rho u_{j} Y}{\partial x_{j}}=\frac{1}{P e} \frac{\partial}{\partial x_{j}}\left(\rho \frac{\partial Y}{\partial x_{j}}\right),
$$

where

$$
P e \equiv \frac{U \ell}{\mathscr{D}}=\operatorname{ReSc}
$$

is the scaling Péclet number (for mass diffusion). This formulation is appropriate for an ideal gas, for example, in the limit of $k_{\mathrm{B}} T$ large compared to the difference in potential energy of molecules at the top and bottom of the flow domain.

The fluids are chosen with matched viscosities, i.e. $\mu_{1}=\mu_{2}=\mu=\bar{\mu}(6 d, e)$, and therefore have different kinematic viscosities, i.e. $v_{2}=\mu_{2} / \rho_{2}=v_{1} / R$. Choosing $\mathscr{D}=\mu / \rho_{1}=v_{1}=v$, we then also have $S c=1$, approximating gas-phase mixing. The scaling Péclet number $(12 b)$ is then equal to the scaling Reynolds number.

Anticipating late-time spatial-resolution requirements, the dimensional value of $\mu$ is chosen so as to yield a scaling Reynolds number given by

$$
R e=\frac{\rho_{1} \ell U}{\mu}=\left(\frac{\ell}{\Delta_{x y}}\right)^{4 / 3},
$$

with $\Delta_{x y}=L / N_{x y}=2 \pi \ell / N_{x y}$, the horizontal grid spacing, and $N_{x y}=256$ the number of grid points along each horizontal direction. With these choices, the (fixed) scaling Reynolds number is equal to $R e \simeq 140.2$ and the outer-scale Reynolds number ( $6 d$ ) is given by

$$
R e_{h}=\frac{\bar{\rho} \dot{h} h}{\mu}=4 \pi \operatorname{Re} \frac{\dot{h}}{U} \frac{h}{L} .
$$

The specific-volume (9), continuity (10), and species-transport (12) equations imply a divergent velocity field (Sandoval 1995),

$$
\nabla \cdot \boldsymbol{u}=-\frac{1}{P e} \nabla \cdot\left(\frac{1}{\rho} \nabla \rho\right) .
$$

Expanding the second term in (10) and combining with (15) yields a non-zero 
(a)

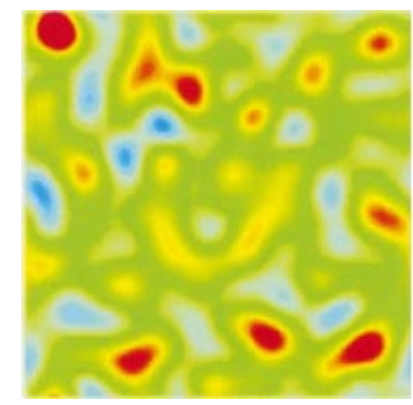

(b)

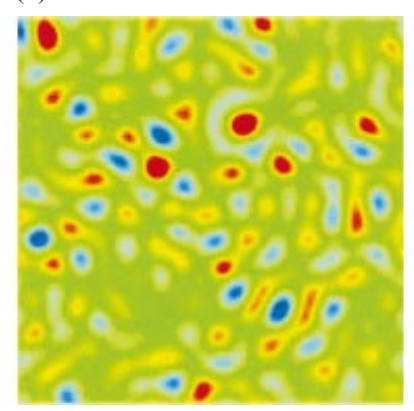

(c)

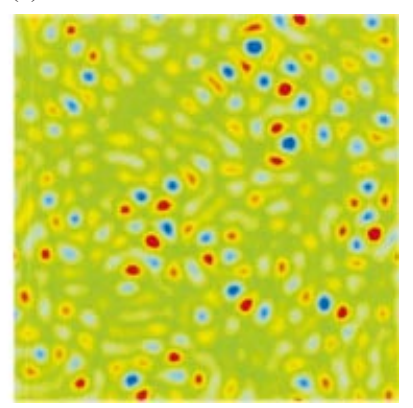

FIGURE 1. Initial interfacial perturbation field (18), $\zeta(x, y)$, for Cases A $(a), \mathrm{B}(b)$, and $\mathrm{C}(c)$. Deep blue: $X \leqslant 0.35$; green: $X=0.5$; red: $X \geqslant 0.65$.

dilatation field, i.e.

$$
\frac{\partial \rho}{\partial t}=-\boldsymbol{u} \cdot \nabla \rho+\frac{\rho}{P e} \nabla \cdot\left(\frac{1}{\rho} \nabla \rho\right) \equiv \Upsilon(\boldsymbol{x}, t),
$$

despite the incompressibility of the flow, a consequence of diffusive mixing of the two, unequal-density species.

\subsection{Boundary and initial conditions}

Periodic boundary conditions are applied in $x$ and $y$, with no-slip walls at the top and bottom $z$-boundaries. With $\boldsymbol{u}=0$ and $\nabla \rho=0$ (no mixed fluid) along the top and bottom walls, the $z$-momentum equation yields the Neumann condition on wall pressure,

$$
\frac{\partial p}{\partial z}=\frac{1}{3 R e} \frac{\partial^{2} w}{\partial z^{2}}-\rho
$$

The implementation of this boundary condition is discussed in Appendix A.

The mole-fraction field $(9 a)$ is initialized as an error function, i.e.

$$
X(x, y, z ; t=0)=\frac{1}{2}\left\{1+\operatorname{erf}\left[\frac{z}{5 \Delta_{z}}+\zeta(x, y)\right]\right\}
$$

where $\Delta_{z}$ is the grid spacing in $z$ and $\zeta(x, y)$ is the perturbation field. To accommodate the unequal bubble and spike growth, the unperturbed origin $(z=0)$ of the initial intermediate $(X=1 / 2)$ isosurface is placed $H / 32$ above the midheight plane, where $H$ is the domain height.

The $\zeta(x, y)$ perturbation field is generated as a two-dimensional field of random numbers, filtered to impose periodicity, transformed to Fourier space, and then Gaussian-filtered before transforming back to physical space. The filter is applied to fit the perturbations to a prescribed spectrum. To satisfy (15), the initial velocity field is prescribed as

$$
\boldsymbol{u}=-\frac{1}{P e} \frac{\nabla \rho}{\rho}
$$

resulting in small initial velocity perturbations in the interfacial region. This is the minimum initial velocity perturbation that must be included for consistency, in the sense that an arbitrary divergence-free velocity field could also be superposed.

Three simulations, Cases A, B, and C, were performed, each with a different initial $\zeta(x, y)$ perturbation, but otherwise identical in every other respect. The two- 


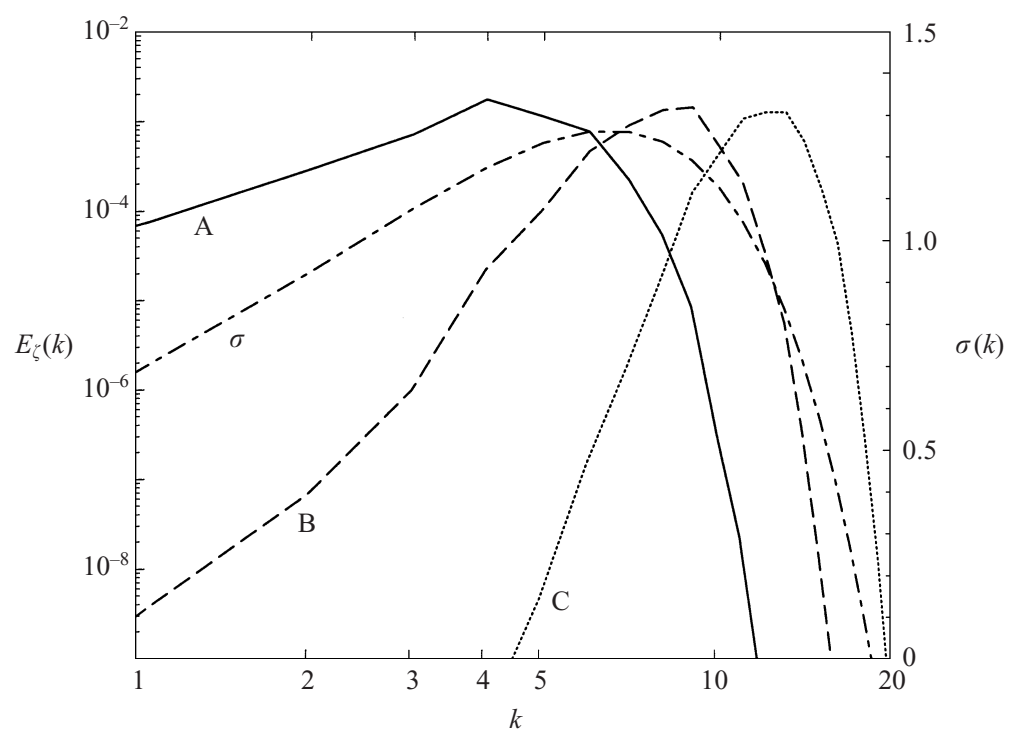

FIGURE 2. Spectra (left axis) of initial interfacial perturbations (18) for Cases A, B, and C, with $k$ denoting the number of waves within $L$. The exponential growth coefficient (right axis), $\sigma(k)$, with units of inverse time, from viscous and diffusive linear-stability theory (Duff et al. 1992) is also plotted.

dimensional perturbation fields, $\zeta(x, y)$, are depicted in figure 1 . They correspond to initial perturbations of decreasing spatial scale, for Cases A, B, and C, respectively.

The corresponding initial perturbation spectra are plotted in figure 2, along with the exponential growth coefficient from viscous and diffusive linear-stability theory (Duff et al. 1962). The wavenumber $k$ in figure 2 denotes the number of waves in the flow-domain width, $L$, i.e. $k=2 \pi /(\lambda / \ell)=L / \lambda$, in the notation of $(6 a, b)$ and the nondimensionalization of (10) and (11). Scaling the linear-stability theory wavenumbers, the growth rate factor is negative for $k>19$, with associated initial perturbation modes expected to be initially damped.

The perturbation energy (interface displacement amplitude squared) is the same in all three cases, in particular

$$
\begin{aligned}
\int_{k_{\mathrm{m}}^{2}} E_{2, \zeta}\left(k_{x}, k_{y}\right) \mathrm{d} k_{x} \mathrm{~d} k_{y} & =\frac{1}{(2 \pi)^{2}} \int_{(2 \pi)^{2}} \zeta(x, y)^{2} \mathrm{~d} x \mathrm{~d} y \\
& =\left\langle\zeta^{2}\right\rangle_{x y}=0.5 \times 10^{-2} \simeq \int_{0}^{k_{\mathrm{m}}} E_{\zeta}(k) \mathrm{d} k
\end{aligned}
$$

where $k=\sqrt{k_{x}^{2}+k_{y}^{2}}$ is the radial wavenumber and $E_{\zeta}(k)$ is the initial radial autospectrum of $\zeta(x, y)$. With 256 points in each of the $(x, y)$-plane directions, the maximum number of waves that can be supported is $k_{\mathrm{m}}=127$. In dimensional units, the magnitude of the perturbation r.m.s., for each case, is $0.0707 \ell \simeq 0.0113 L$. Defining the profile thickness, $h=z_{2}-z_{1}$, in terms of the $1 \%$ profile points, i.e. $X\left(z_{1}\right)=0.01$ and $X\left(z_{2}\right)=0.99$, the (unperturbed) initial error-function thickness is given by $h_{0} \simeq 16.5 \Delta_{z}$, cf. (18). For all three cases, the ratio of the initial r.m.s.-based perturbation amplitude 
to interface thickness is

$$
\frac{\zeta^{\prime}}{h_{0}} \simeq 0.28
$$

If, for scaling purposes, $k_{\mathrm{c}} \equiv L / \lambda_{\mathrm{c}}$ in $(6 a, b)$ is identified with the peak of the perturbation spectrum, then $k_{\mathrm{c}} \simeq 4,9$, and 12.5 for Cases $\mathrm{A}, \mathrm{B}$, and $\mathrm{C}$, respectively, with modal contributions extending to roughly twice these values in each case (cf. figures 1 and 2).

\section{Solution technique}

\subsection{Spatial discretization}

To capture the full range of length scales in this flow, high-order, high-resolution numerical methods are employed, so that numerical dissipation does not compete with physical dissipation. For example, mixing, represented by the diffusion term in (12), involves second derivatives and is sensitive to high wavenumbers.

The simulations are performed on a mesh of $256 \times 256 \times 1024$ grid points in $x$, $y$ and $z$, respectively, with $x$ - and $y$-derivatives computed spectrally. Derivatives in $z$ are computed with an eighth-order compact scheme (Lele 1992). The grid spacing in $z$ is reduced, relative to the spacing in $x$ and $y$, to account for the difference in resolving power between the Fourier-spectral and the compact methods (Appendix B). This leads to the choice, $\Delta_{z}=8 \Delta_{x y} / 13$, yielding a flow-domain aspect ratio of $H / L=32 / 13$.

\subsection{Temporal discretization}

Time integration of (16) is via the third-order Adams-Bashforth-Moulton (ABM) method. The predictor and corrector steps are

$$
\rho^{*}=\rho^{n}+\frac{1}{2} \Delta t\left(3 \Upsilon^{n}-\Upsilon^{n-1}\right),
$$

and

$$
\rho^{n+1}=\rho^{*}+\frac{5}{12} \Delta t\left(\Upsilon^{*}-2 \Upsilon^{n}+\Upsilon^{n-1}\right),
$$

respectively. Here $n=t / \Delta t$ denotes the integer timestep and an asterisk denotes a predicted value at the $n+1$ timestep. Before (23) can be computed, $\Upsilon^{*}$ must first be obtained, which requires that (11) be advanced by the predictor step.

A pressure-projection scheme for the momentum equation is derived by integrating (11) from $t$ to $t+\Delta t$, i.e.

$$
\left(\rho u_{i}\right)^{n+1}=\left(\rho u_{i}\right)^{n}+\int_{t}^{t+\Delta t} \Lambda_{i} \mathrm{~d} t-\Delta t\left(\frac{\partial p_{\mathrm{a}}}{\partial x_{i}}+\rho_{\mathrm{a}} \delta_{i 3}\right),
$$

where

and

$$
\begin{gathered}
\Lambda_{i} \equiv \frac{\partial}{\partial x_{j}}\left(\tau_{i j}-\rho u_{i} u_{j}\right), \\
p_{\mathrm{a}} \equiv \frac{1}{\Delta t} \int_{t}^{t+\Delta t} p \mathrm{~d} t
\end{gathered}
$$

$$
\rho_{\mathrm{a}} \equiv \frac{1}{\Delta t} \int_{t}^{t+\Delta t} \rho \mathrm{d} t=\frac{1}{2}\left(\rho^{n+1}+\rho^{n}\right)+O\left(\Delta t^{2}\right) .
$$


Equation (24) is split into two parts. The first,

$$
\Phi_{i}=\left(\rho u_{i}\right)^{n}+\int_{t}^{t+\Delta t} \Lambda_{i} \mathrm{~d} t
$$

accounts for advection and diffusion, while the second,

$$
\left(\rho u_{i}\right)^{n+1}=\Phi_{i}-\Delta t\left(\frac{\partial p_{\mathrm{a}}}{\partial x_{i}}+\rho_{\mathrm{a}} \delta_{i 3}\right),
$$

accounts for pressure and acceleration.

Equations (25) and (26) are advanced as follows. In the predictor step, $\Phi_{i}$ is computed using the Adams-Bashforth approximation,

$$
\int_{t}^{t+\Delta t} \Lambda_{i} \mathrm{~d} t=\frac{1}{2} \Delta t\left(3 \Lambda_{i}^{n}-\Lambda_{i}^{n-1}\right)+O\left(\Delta t^{3}\right)
$$

At this point, $p_{\mathrm{a}}$ is needed for (26). Taking the divergence of (26) yields the Poisson equation,

$$
\frac{\partial^{2} p_{\mathrm{a}}}{\partial x_{i}^{2}}=-\frac{1}{\Delta t}\left[\frac{\partial}{\partial x_{i}}\left(\rho u_{i}\right)^{n+1}-\frac{\partial \Phi_{i}}{\partial x_{i}}\right]-\frac{\partial \rho_{\mathrm{a}}}{\partial z} .
$$

Since $\left(\rho u_{i}\right)^{n+1}$ is unknown at this point, $\partial\left(\rho u_{i}\right)^{n+1} / \partial x_{i}$ is approximated by expanding and combining with (15), i.e.

$$
\frac{\partial}{\partial x_{i}}\left(\rho u_{i}\right)^{n+1}=u_{i}^{n+1} \frac{\partial \rho^{n+1}}{\partial x_{i}}-\frac{\rho^{n+1}}{\operatorname{ReSc}} \frac{\partial}{\partial x_{i}}\left(\frac{1}{\rho^{n+1}} \frac{\partial \rho^{n+1}}{\partial x_{i}}\right),
$$

and extrapolating $u_{i}^{n+1}$ from previous timesteps, i.e.

$$
u_{i}^{n+1}=2 u_{i}^{n}-u_{i}^{n-1}+O\left(\Delta t^{2}\right) .
$$

In this first step, $\rho^{n+1}$ is approximated using $\rho^{*}$. With these substitutions, (27) is solved for $p_{\text {a }}$ (see Appendix A) which is then substituted into (26) to compute $\left(\rho u_{i}\right)^{*}$, the predicted value of $\left(\rho u_{i}\right)^{n+1}$.

In the corrector step, $u_{i}^{*}$ and $\Upsilon^{*}$ are computed from $\rho^{*}$ and $\left(\rho u_{i}\right)^{*}$, and $\rho^{n+1}$ is obtained from (23). Next, (25) is computed using the trapezoidal rule, i.e.

$$
\int_{t}^{t+\Delta t} \Lambda_{i} \mathrm{~d} t=\frac{1}{2} \Delta t\left(\Lambda_{i}^{*}+\Lambda_{i}^{n}\right)+O\left(\Delta t^{3}\right)
$$

Equation (27) is now solved using $\left(\rho u_{i}\right)^{*}$ in place of $\left(\rho u_{i}\right)^{n+1}$. No approximation is necessary for $\rho^{n+1}$, which is now known. Finally, (26) is used to compute $\left(\rho u_{i}\right)^{n+1}$, from which $u_{i}^{n+1}$ is obtained. Time integration is initiated using the Matsuno (1966) method for the first timestep (cf. Durran 1991), i.e. a first-order predictor and corrector are used instead of (22) and (23), with the first-order extrapolation, $u_{i}^{n=1}=u_{i}^{n=0}$, for the velocity.

\section{Results}

\subsection{Verification}

The code was verified to conserve mass and momentum, as well as preserve symmetry in separate tests with symmetric initial and boundary conditions.

Numerical results for the growth of small-amplitude, single-mode perturbations were tested against predictions of linear stability theory (LST). A comparison is 


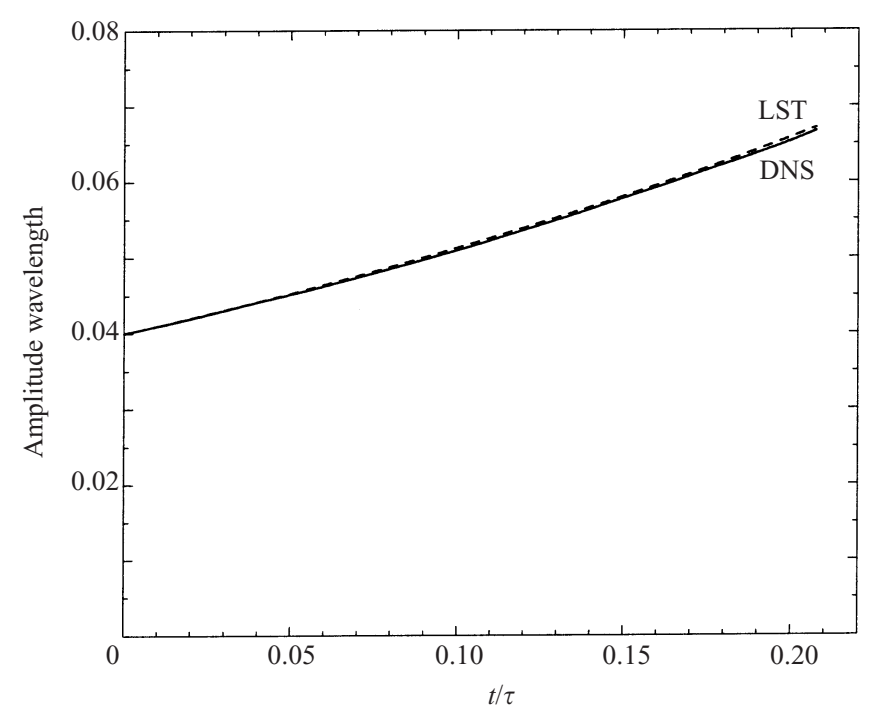

FIGURE 3. Comparison of direct numerical simulation (DNS, solid line) with linear-stability theory (LST, dashed line) for a single-mode, two-dimensional, small-amplitude perturbation.

shown in figure 3 , using $\tau$ as the scaling time $(6 b, c)$, whose non-dimensional value is $\tau=\sqrt{2 \pi / \mathscr{A}}$ and which, for the $\mathscr{A}=1 / 2$ case investigated here, is $\tau=\sqrt{4 \pi}$. The comparison was performed in the low- $k$ regime where viscous and diffusive contributions are negligible. The $z$-grid spacing was reduced by a factor of 4 , compared to the $x$-grid spacing, to decrease the density interface thickness relative to the perturbation amplitude. The thickness correction, $\psi$, in the Duff et al. (1962) notation, was determined to decrease the growth rate by about $1 \%$; this was factored into the LST curve. The agreement between theory and simulation is very good until amplitudes become weakly nonlinear, causing the LST prediction to slightly exceed the simulated growth rate.

To test species-diffusion effects, early-time results were compared to theoretical predictions for the diffusive evolution of the initial mole-fraction profile (18). In the limit of $\zeta(x, y) \rightarrow 0$ and the units of (12), purely diffusive evolution leads to

$$
X(z)=\frac{1}{2}\left\{1+\operatorname{erf}\left[\frac{z}{2 \sqrt{\left(t+t_{0}\right) / P e}}\right]\right\},
$$

with $\sqrt{t_{0} / P e}=5 \Delta_{z} / 2$, as required to match the profile thickness at $t=0$. Measuring the profile thickness, $h$, in terms of the $1 \%$ points (21), this predicts

$$
h_{\mathrm{th}}(t) \simeq 6.58 \sqrt{\left(t+t_{0}\right) / P e} .
$$

The steep initial mole-fraction profiles lead to rapid diffusive growth, which dominates overall growth at early times, resulting in a simulated mixing-zone evolution wellapproximated by (Case $\mathrm{C}$ data)

$$
h_{\text {sim }}(t) \simeq 6.6 \sqrt{\left(t+t_{0}\right) / P e},
$$

in good agreement with theory. Growth during the diffusive-growth stage is the same for all cases (independent of initial perturbations), as will be discussed below. 

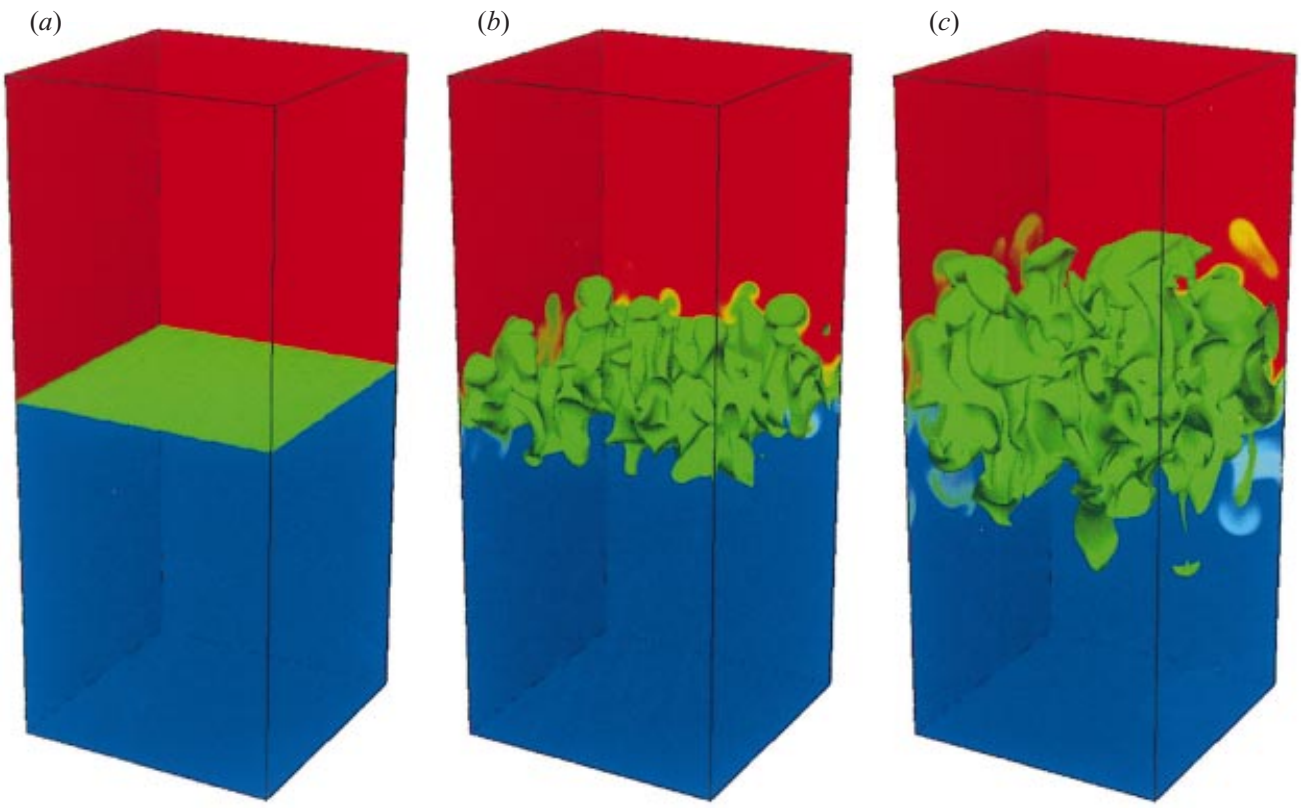

Figure 4. Time-evolution of intermediate $(X=0.5)$-isosurface (green) for Case C. Pure heavy fluid $(X=1)$ is red, pure light fluid $(X=0)$ is blue. Times for the three images displayed: $(a) t / \tau=0$, (b) 3.44, and (c) 4.63 .

\subsection{Diffusive and nonlinear stages}

\subsubsection{Visualization}

As mentioned previously, three simulations were carried out: Cases A, B, and C. The evolution of the density field for Case $\mathrm{C}$ is depicted in 4 , which is initiated with the maximum number of waves. Pure heavy fluid $(X=1)$ is red, pure light fluid $(X=0)$ is blue, and the intermediate $(X=0.5)$-isosurface $(1: 1$ molar ratio) is green. In $(a)$ $(t / \tau=0)$, the initial perturbations are just barely discernible on the interface. In $(b)$ $(t / \tau=3.44)$, mushroom-like structures are visible on the upper side of the isosurface. In $(c)(t / \tau=4.63)$, the original mushrooms have merged into larger structures and a long, thin protuberance ('spike') can be seen penetrating deep into the light fluid. The neck of this structure has been pinched off by diffusion. The diffusion term in (12) is responsible for altering the topology of the $(X=0.5)$-isosurface; absent this term, all isopycnals would remain simply connected.

\subsubsection{Mixing-zone growth}

To measure mixing-zone growth, the mole-fraction field $(9 a)$ is averaged in the homogeneous directions, i.e.

$$
\langle X(\boldsymbol{x}, t)\rangle_{x y} \equiv \frac{1}{(2 \pi)^{2}} \int_{(2 \pi)^{2}} X(\boldsymbol{x}, t) \mathrm{d} x \mathrm{~d} y .
$$

Figure 5 displays the horizontally averaged mole-fraction profile for Case $\mathrm{C}$, at five different times. The profile retains the initial error-function shape, for some time, in a manner that belies the complexity of the underlying flow structure. As discussed below, it subsequently evolves to a different shape and eventually exhibits irregular features, a consequence of averaging over fewer, larger-scale structures.

The penetration length of the spikes and bubbles, $h_{\mathrm{s}}(t)$ and $h_{\mathrm{b}}(t)$, respectively, are 


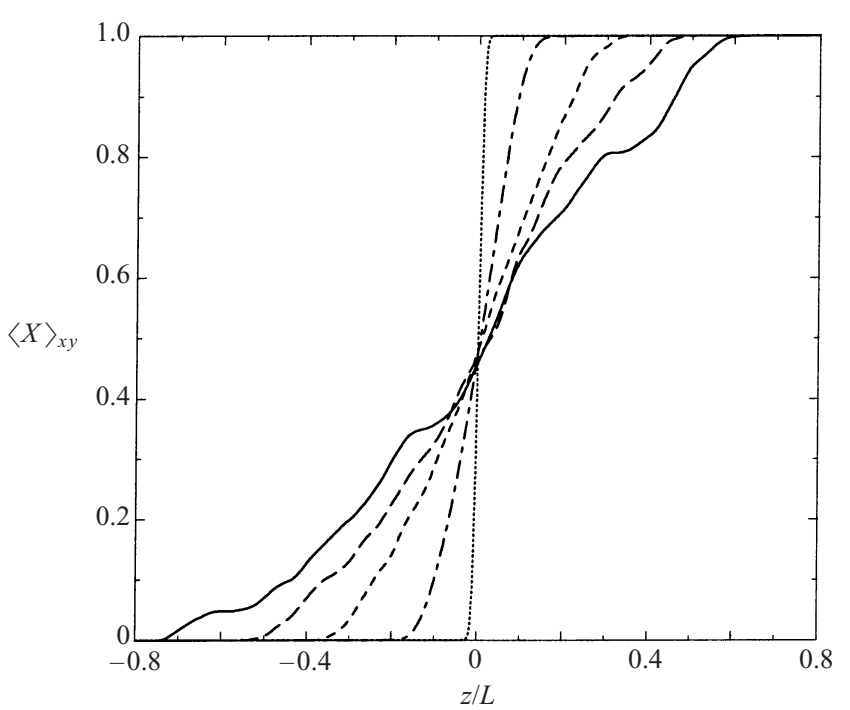

FIGURE 5. Time-evolution of horizontally averaged mole-fraction field for Case C. Dotted line: $t / \tau=0$, short-long dashed line: $t / \tau=2.26$, short-dashed line: $t / \tau=3.40$, long-dashed line: $t / \tau=3.95$, solid line: $t / \tau=4.52$.

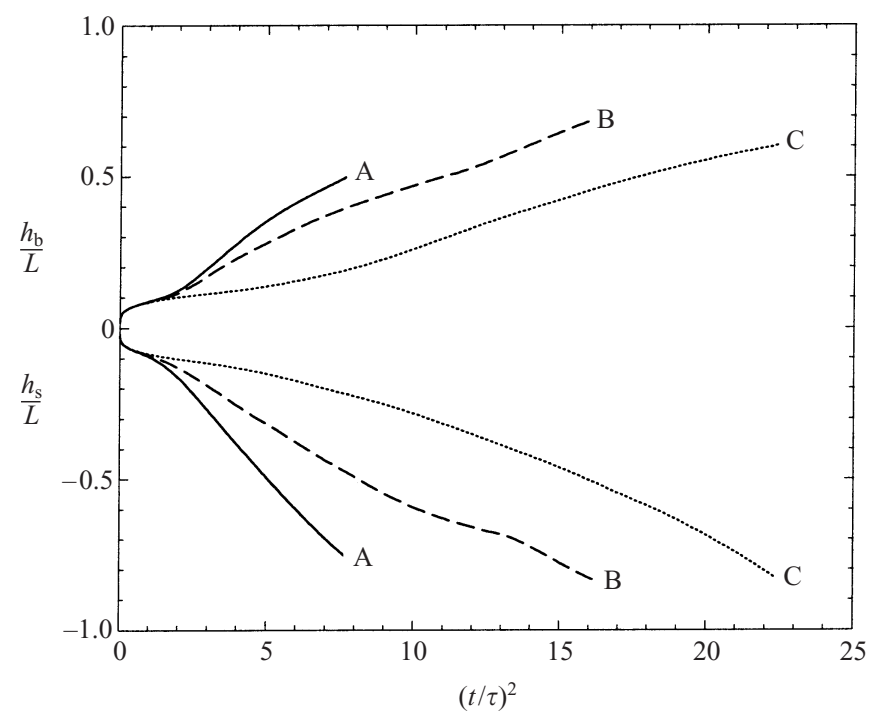

Figure 6. Penetration distance of bubbles $\left(h_{\mathrm{b}} / L\right)$ and spikes $\left(h_{\mathrm{s}} / L\right) v s .(t / \tau)^{2}$.

defined as the $z$-distances for which $\langle X(x, t)\rangle_{x y} \geqslant \epsilon$ and $\langle X(\boldsymbol{x}, t)\rangle_{x y} \leqslant 1-\epsilon$, respectively, with $\epsilon=0.01$. This criterion for profile thickness was used in providing a verification comparison with diffusive-growth predictions, as discussed above. It is also commonly used in boundary-layer analysis, as a marker of velocity-profile edges, as well as for the analysis of shear-layer growth and mixing (e.g. Dimotakis 1991), permitting direct comparisons between these two flows, as discussed below. The initial intermediate isosurface $(X=1 / 2)$ is placed at $z=0$; cf. (18).

The resulting bubble and spike penetrations are plotted in figure $6, v s .(t / \tau)^{2}$, to facilitate comparison with the growth models described in the Introduction. Growth 


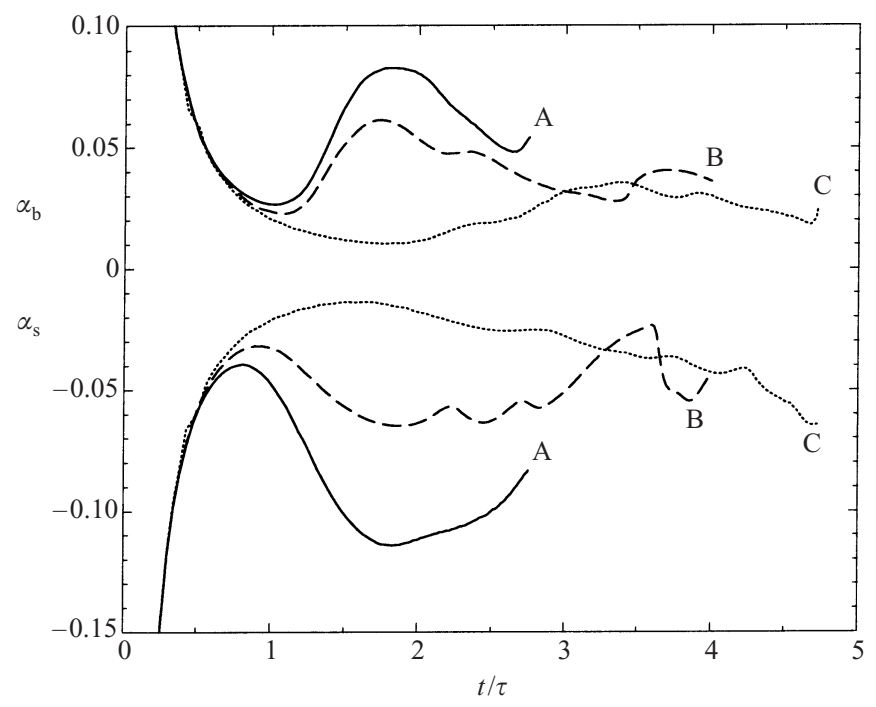

FIGURE 7. Growth coefficients for the three cases: $\alpha_{\mathrm{b}}$ for 'bubbles' (positive values) and $\alpha_{\mathrm{s}}$ for 'spikes' (negative values).

rates can be seen to sensitively depend on the initial conditions. Case A, initialized with perturbations encompassing the fewest waves $\left(k_{\mathrm{c}} \simeq 4\right)$, grows fastest. Progressively slower growth is found for Cases B and $\mathrm{C}\left(k_{\mathrm{c}} \simeq 9\right.$ and 12.5 , respectively). The values for $(t / \tau)^{2}$ required to attain the same $h_{i} / L$ differ by a factor of 3 between Cases $\mathrm{A}$ and $\mathrm{C}$. The Duff et al. linear-stability analysis might have suggested that Case $\mathrm{B}$, subtending the greatest mean-square amplitude under the instability curve, $\sigma(k)$, should have exhibited the highest growth rate.

The departure of the bubble-/spike-front growth from a quadratic time dependence can be assessed by computing the derivative of $h_{\mathrm{b}} / L$ and $h_{\mathrm{s}} / L$ with respect to $(t / \tau)^{2}$, permitting a comparison with the $\alpha_{i}$ model parameters in (1) and (4). The results are depicted in figure 7. The $\alpha_{i}$ exhibit substantial differences between cases and are not well-approximated by constants. No doubt, the variations must be attributed, in part, to the fact that only a few bubble/spike structures are responsible for defining the $1 \%$ front, and hence the value of the $\alpha_{i}$. Nevertheless, the variations are large. For Case B, the $\alpha_{i}$ decrease in absolute value for $t / \tau \gtrsim 1.5$, corresponding to overall growth slower than quadratic. For Case C, which is initialized by a large number of waves (recall figure 1) and exhibits the smallest fluctuations, their difference approaches a constant, i.e. overall growth nearly quadratic, but with (algebraically) decreasing individual $\alpha_{i}$ for $t / \tau \gtrsim 3$, i.e. non-quadratic individual front advance.

Figures 6 and 7 also illustrate the slightly asymmetric bubble/spike evolution at this Atwood number. Penetration of the high-density fluid into the low-density fluid exceeds that of the low-density fluid into the high-density fluid, as noted previously (e.g. Read 1984, in experiments with $\rho_{2} / \rho_{1}=3$, reports $h_{\mathrm{s}} / h_{\mathrm{b}} \approx 1.3$ ). If the overall mixing zone is defined as bounded by the $1 \%$ limits of the horizontally averaged mole-fraction profiles, it is seen to be descending into the light fluid. Slightly more low-density than high-density fluid is entrained into the RTI mixing zone.

Figure 8 depicts the overall extent, $h(t)$, of the RTI mixing zone $(3 a)$, along with indicatrices corresponding to $t^{1 / 2}$ and $t^{2}$ growth. Growth during the diffusiondominated, $h \propto t^{1 / 2}$, regime is seen to be the same for all cases, i.e. independent 


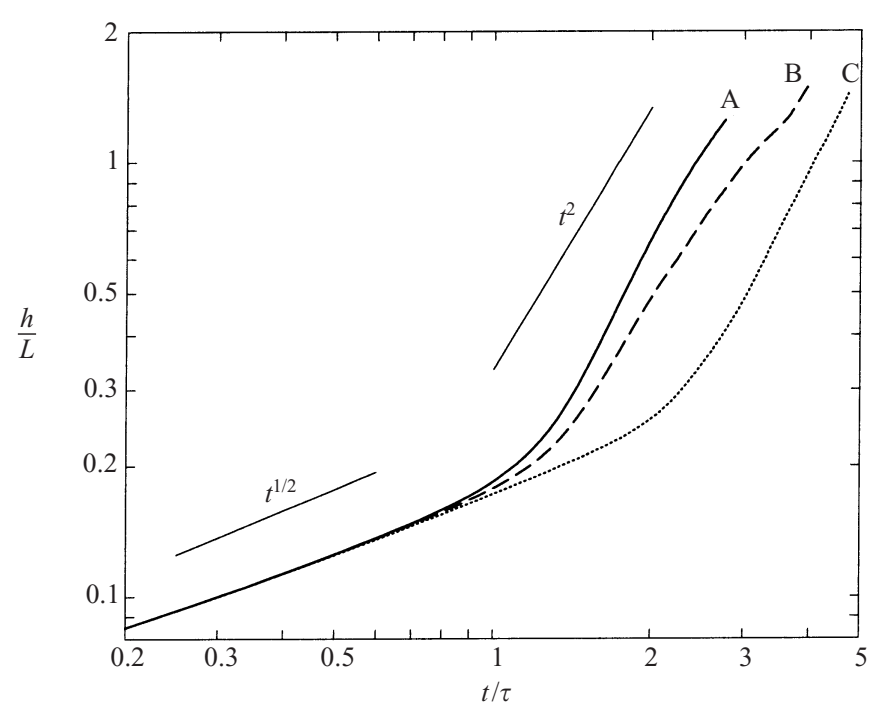

FIGURE 8. Mixing-zone extent, $h / L=\left(h_{\mathrm{b}}-h_{\mathrm{s}}\right) / L$, vs. $t / \tau$ for the three cases.

of the initial perturbations, and in good agreement with analytical predictions for purely diffusive growth (recall discussion in $\S 4.1$ ). The onset of a faster-growth stage is conspicuous, where growth is faster than diffusive. This occurs at a different time for each of the three cases, reflecting differences in the initial modal seeding in each case.

Despite the significant variation in the $\alpha_{i}$ (cf. figure 7), figure 8 confirms that overall growth in the post-diffusive regime is well-represented as quadratic for Case C. However, it is difficult to say whether deviation from quadratic growth for Cases A and $\mathrm{B}$ is because of inadequate statistical convergence, because asymptotic growth is not quadratic, or because asymptotic behaviour has not been attained, e.g. because initial modal seeding $\left(k_{\mathrm{c}}\right)$ is too low for those cases. If overall growth is to be approximated as quadratic, with departures attributed to statistical reasons, the results indicate that the effective growth coefficients, $\alpha$, are sensitive functions of the initial conditions.

\subsubsection{Mole-fraction profiles}

The evolution of the $\langle X(z, t)\rangle_{x y}$ profiles can be assessed by plotting them with respect to a scaled vertical coordinate, $z / h(t)$. Averaged profiles from the three cases, scaled in this fashion, are depicted in figures 9,10 , and 11.

The mole-fraction profiles transition from their initial error-function shape, retained during most of the $h \propto t^{1 / 2}$ stage, to a profile that is slightly asymmetric, i.e. with a small deviation from antisymmetry. This is illustrated by the scaled profiles for Cases A (figure 9) and B (figure 10).

The results indicate an approach to self-similarity, if the vertical coordinate is scaled by the mixing-zone height, $h(t)$. This is in accord with previous experimental findings (e.g. Kucherenko et al. 1997). The small deviations from such a collapse and self-similarity are probably attributable to statistical-convergence difficulties, as alluded to above. However, the late-time quasi-self-similar profiles are different for each of the three cases, with profiles for Case A deviating the most from their initial shape and less so for Cases B and C. 


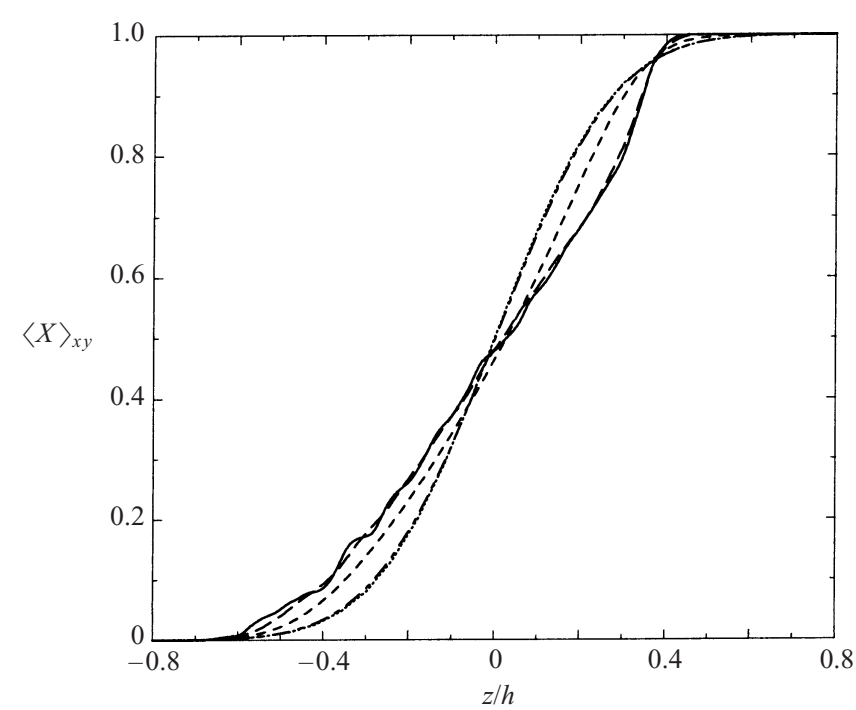

FIGURE 9. Time-evolution of horizontally averaged mole-fraction profiles for Case A. Dotted line: $t / \tau=0$, short-long dashed line: $t / \tau=0.639$, short-dashed line: $t / \tau=1.236$, long-dashed line: $t / \tau=1.859$, solid line: $t / \tau=2.432$.

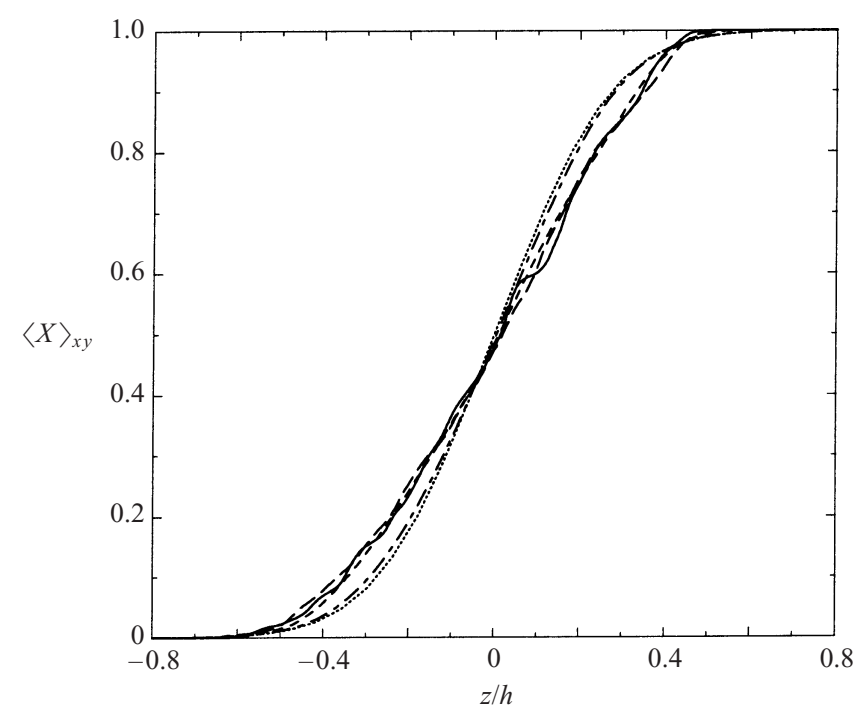

FIGURE 10. Time-evolution of horizontally averaged mole-fraction profiles for Case B. Dotted line: $t / \tau=0$, short-long dashed line: $t / \tau=0.852$, short-dashed line: $t / \tau=1.711$, long-dashed line: $t / \tau=2.541$, solid line: $t / \tau=3.036$.

\subsubsection{Reynolds numbers}

An important measure of growth and independence from viscous/diffusive effects is the outer-scale Reynolds number $(6 d)$, (14). These are plotted in figure 12 for each case. All cases achieve maximum outer Reynolds numbers over 3000. Case C evolves most slowly, allowing the interpenetrating fluids more time to diffuse before strong straining ensues. This places less stringent simulation requirements vis-à-vis aliasing limits, permitting Case C to achieve a slightly higher Reynolds number (3700) than 


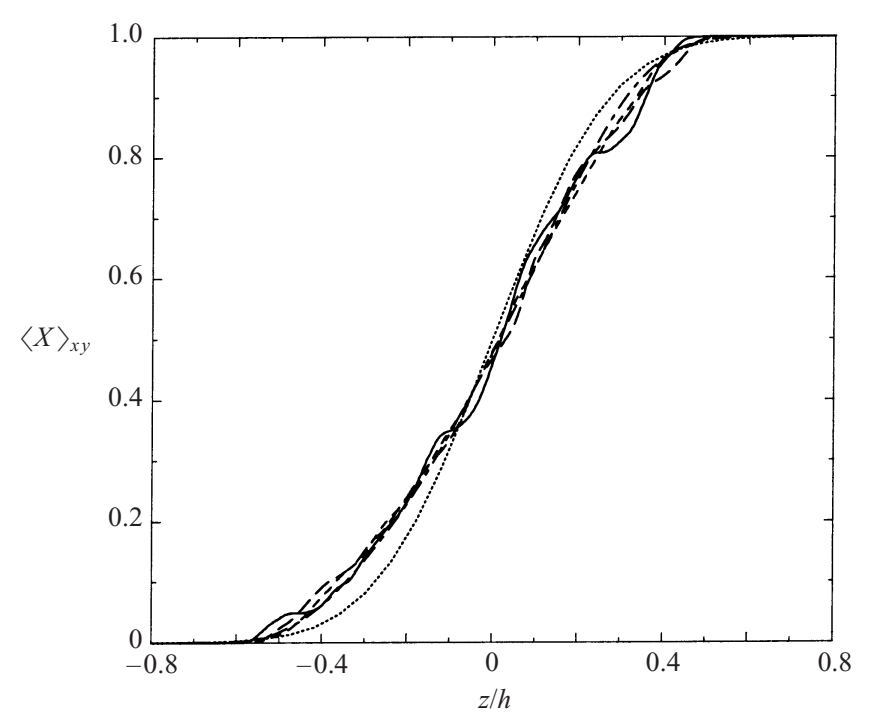

FIGURE 11. Time-evolution of horizontally averaged mole-fraction profiles for Case C. Line type legend as in figure 5.

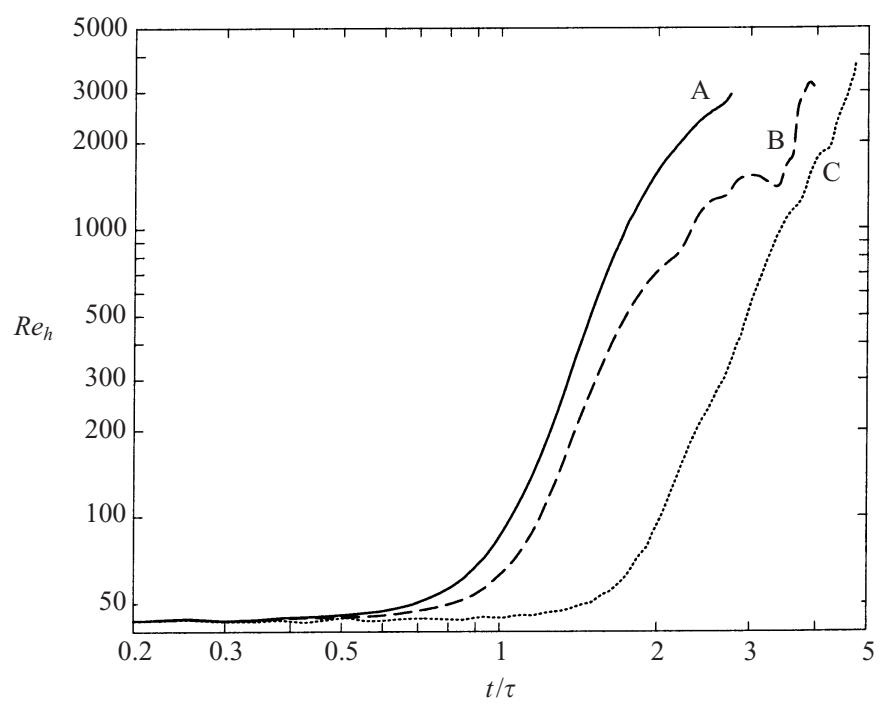

FigURE 12. Outer-scale Reynolds number, $R e_{h} \equiv \bar{\rho} h \dot{h} / \mu$, vs. $t / \tau$, for the three cases.

Cases A or B. The non-monotonic curve for Case B is a consequence of the definition of $\dot{h}$, which is sensitive to the leading bubble, or spike. $\dagger$ This can lead to a momentary 'retrograde' mixing-zone advance, as illustrated in the plot of $\alpha_{\mathrm{s}}$ for Case B in figure 7.

As figure 8 suggests, time is not the most appropriate progress variable for Rayleigh-Taylor mixing-zone dynamics. A particular mixing-zone height, $h(t)$, is attained at rather different times depending on initial conditions. Figure 13 depicts outer Reynolds numbers $v s . h / L$ and corroborates the general finding that $h(t)$ is to be

$\dagger$ Diffusive mixing can decrease the density difference across such a front and either decrease its advance velocity (4), to be overtaken by another bubble or spike, or decrease its contribution to the mole-fraction profile, resulting in a different leading feature marking the $1 \%$ point. 


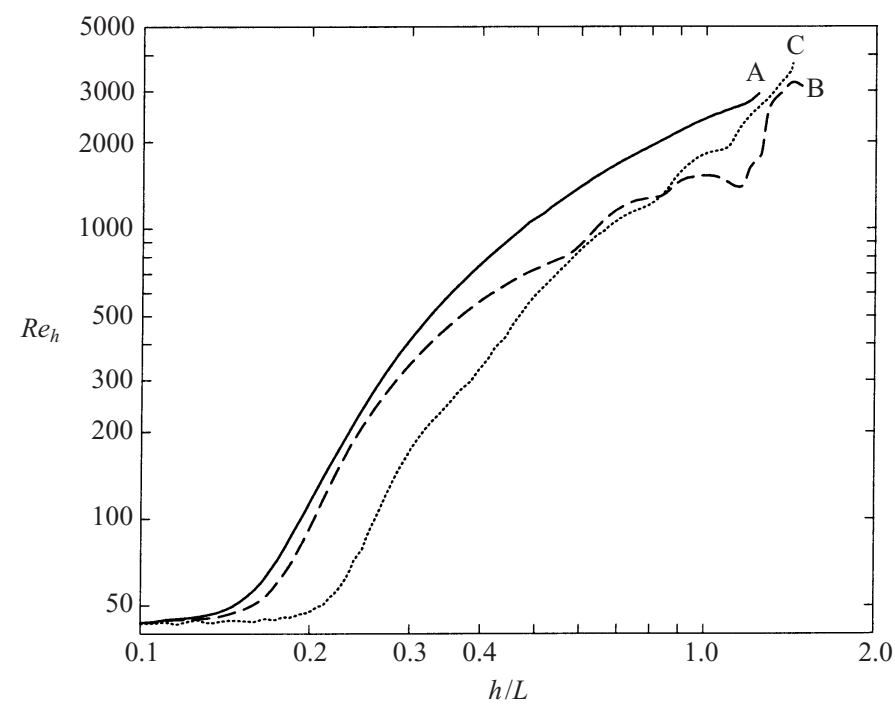

FIGURE 13. Outer Reynolds number, $v s . h / L$, for the three cases.

preferred as a progress variable, resulting in improved collapse in the post-diffusive regime, when quantities are scaled in terms of it. Interestingly, $\operatorname{Re}_{h}(h)$ for Case $\mathrm{C}$ crosses over that for Case B, whereas the $R e_{h}(t)$ (figure 12) are ordered as for $h$ (figure 8). Since $R e_{h} \propto \dot{h} h$, this also indicates an improved, if imperfect, correlation between mixing-zone height, $h$, and its rate of growth, $\dot{h}(3)$.

\subsection{Mixing}

The preceding analysis focuses on the behaviour of the averaged density field, without regard to local-composition behaviour. Averaged density profiles do not distinguish between unmixed fluid in equal proportions in a particular $z$-plane, for example, for which $\langle X(z, t)\rangle_{x y}=1 / 2$, and fluid mixed on a molecular scale to a 50:50 ratio, for which $X(\boldsymbol{x}, t)=1 / 2$. The distinction is significant since the driving force depends on local density differences (4). Also, molecular mixing and chemical reactions between the two interpenetrating fluids may be of specific interest.

Mixture composition can be expressed in terms of the local mole fraction (9a) and quantified in terms of the amount of chemical product that would result from a fast-kinetic chemical reaction between the two fluids. In particular, the chemical product is limited by the amount of lean reactant, i.e.

$$
X_{\mathrm{p}}\left(X ; X_{\mathrm{s}}\right)= \begin{cases}\frac{X}{X_{\mathrm{s}}} & \text { for } X \leqslant X_{\mathrm{s}} \\ \frac{1-X}{1-X_{\mathrm{s}}} & \text { for } X \geqslant X_{\mathrm{s}},\end{cases}
$$

where $X_{\mathrm{s}}$ is the (heavy-fluid) mole fraction for a stoichiometric mixture. This will be taken as $X_{\mathrm{s}}=1 / 2$ in the analysis below. The total chemical product (thickness) formed in the Rayleigh-Taylor cell is then given by the integral over the cell height, i.e.

$$
P_{\mathrm{t}}=\int_{H}\left\langle X_{\mathrm{p}}(X)\right\rangle_{x y} \mathrm{~d} z
$$




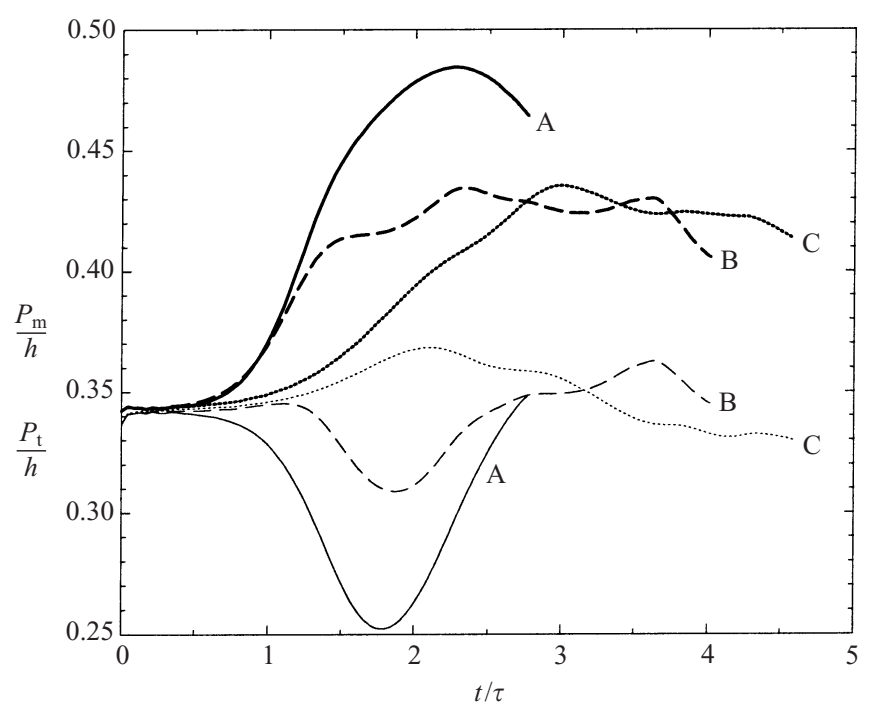

Figure 14. Scaled maximum $\left(P_{\mathrm{m}} / h\right.$; top curves) and total $\left(P_{\mathrm{t}} / h\right.$; bottom curves $)$ chemical product thickness, $v s . t / \tau$, for the three cases.

If all fluid in a particular $z$-plane were mixed, it would have a composition

$$
X(x, y, z)=\langle X(z)\rangle_{x y} .
$$

This can be used to compute the maximum amount of chemical product per unit area that would be formed at $z$ in a fast chemical reaction, as a result of complete mixing/homogenization of the fluid,

$$
\left\langle X_{\mathrm{p}}(X)\right\rangle_{x y}=\frac{1}{(2 \pi)^{2}} \int_{(2 \pi)^{2}} X_{\mathrm{p}}[X(x, y, z)] \mathrm{d} x \mathrm{~d} y .
$$

Similarly, the maximum product (thickness) possible in the cell, resulting from complete homogenization of fluid in each $z$-plane, is given by

$$
P_{\mathrm{m}}=\int_{H} X_{\mathrm{p}}\left(\langle X\rangle_{x y}\right) \mathrm{d} z
$$

The normalized product thicknesses, $P_{\mathrm{t}} / h$ and $P_{\mathrm{m}} / h$, are plotted in figure $14, v s . t / \tau$, for the three cases. As the figure illustrates, there are significant differences between the $h, P_{\mathrm{t}}$, and $P_{\mathrm{m}}$ length scales. The extent, $h$, of the mixing zone is not a good surrogate for either the total product, or the maximum possible product, as defined above. Interestingly, the total chemical product returns to its initial (diffusive) value towards the end of all three simulations, $P_{\mathrm{t}} / h \approx 0.34$. This is very close to values encountered in high Reynolds number, gas-phase, chemically reacting shear layers (Dimotakis 1991, figure $21, P_{\mathrm{t}} / h \rightarrow \delta_{\mathrm{p}}(\xi=0.5) / \delta$ in that notation).

The ratio of the two chemical-product thicknesses,

$$
\Xi=\frac{P_{\mathrm{t}}}{P_{\mathrm{m}}}
$$

measures the total product formed relative to the product that would be formed if all entrained fluid were completely mixed within each $z$-plane. The parameter $\Xi$ provides a mixing metric analogous to the Youngs (1994) 'molecular mixing fraction' 


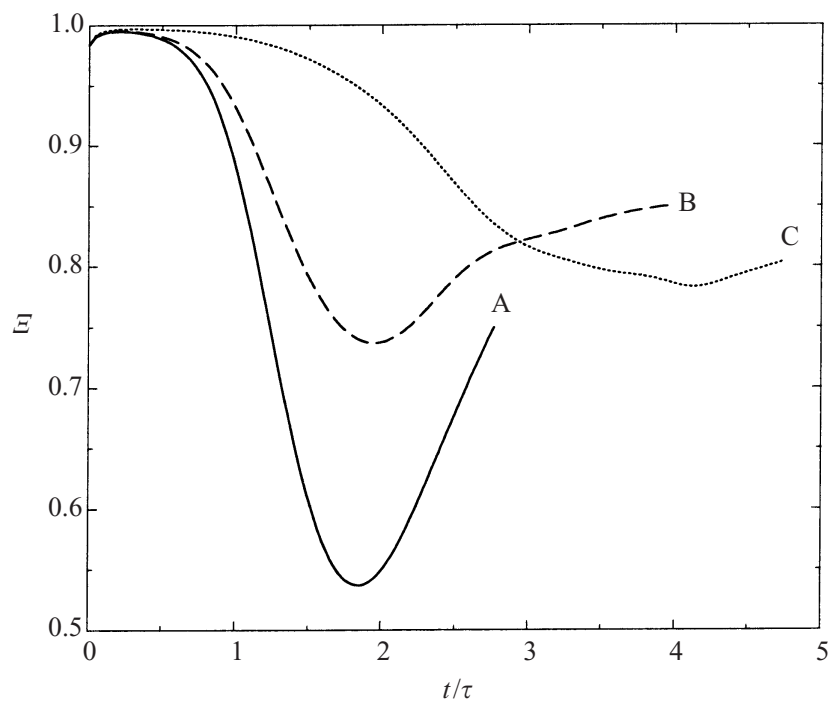

FIGURE 15 . Mixing parameter, $\Xi$ (35), vs. $t / \tau$, for the three cases. $\Xi=1$ implies complete mixing within each $z$-plane (homogenized fluids). $\Xi=0$ implies completely segregated fluids.

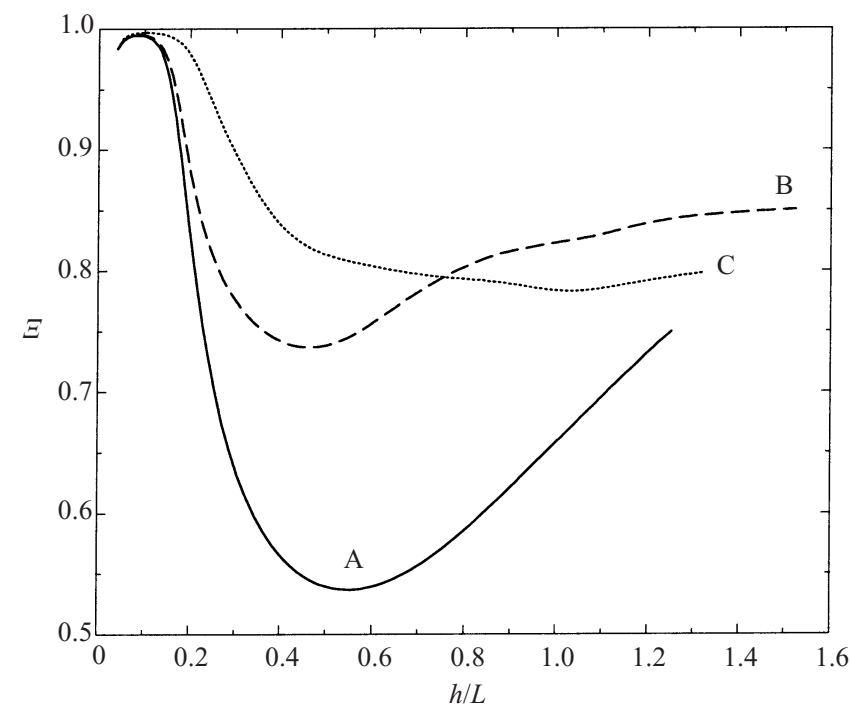

FIGURE 16. Mixing parameter, $\Xi$, vs. $h / L$, for the three cases.

parameter, $\Theta$. The mixing parameter, $\Xi$, is plotted in figure $15 \mathrm{vs.} t / \tau$. Initially, the layers are diffuse, with only small-amplitude perturbations, and $\Xi$ is close to unity in all cases, i.e. fluid within the mixing zone during the diffusive-growth phase may be regarded as completely mixed. Considering differences in initial modal seeding $\left(k_{\mathrm{c}}\right)$ between the three cases, the results support the intuitive notion that fluid entrained as a consequence of large-scale motions (lower surface-to-volume ratio) mixes more slowly than fluid entrained at smaller scales. Hence, mixing for Case A is slowest and mixing for Case $\mathrm{C}$ is fastest (cf. figure 2). Mixing, as measured by $\Xi$, differs substantially between the cases. Comparison in terms of $\Xi(h / L)$ indicates a somewhat 


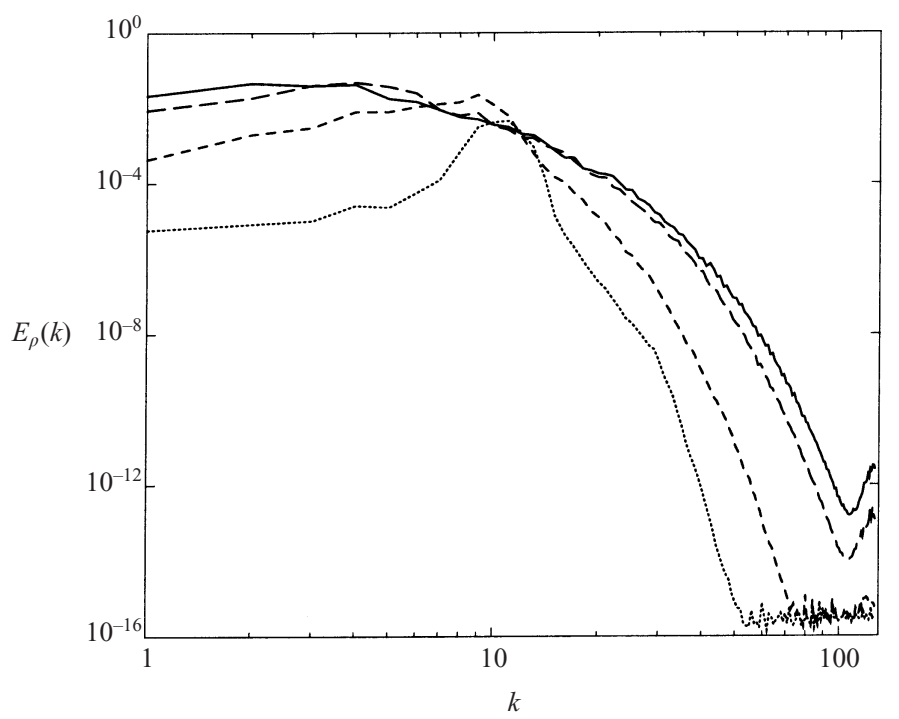

FIGURE 17. Radial autospectra of density fluctuations in the $(z=0)$-plane for Case C. Lines of increasing solidity denote increasing time. Dotted line: $t / \tau=1.15$, short-dashed line: $t / \tau=2.26$, long-dashed line: $t / \tau=3.40$, solid line: $t / \tau=4.52$.

lower variance (figure 16), but the significant differences among cases persist. Mixing appears to be even more sensitive to initial conditions than growth rates. Again, $h / L$ emerges as the preferred progress variable, with all three cases indicating a qualitative change (improved mixing for Cases A and B) at $h / L \simeq 0.5$.

\subsection{Spectra}

The radial autospectra, $E_{\rho}(k)$, of the density fluctuations were computed in the $(z=0)$-plane, $\rho(x, y, z=0)$, defined as was $E_{\zeta}(k)$ for $\zeta(x, y)$ in $(20)$,

$$
\int_{0}^{k_{\mathrm{m}}} E_{\rho}(k) \mathrm{d} k=\left\langle\rho^{2}\right\rangle_{x y}-\langle\rho\rangle_{x y}^{2}=\left\langle\rho^{\prime 2}\right\rangle_{x y},
$$

with $k$ the radial wavevector, as in (20). These are plotted in figure 17 for Case C. As the flow evolves, the peak in the spectrum moves to lower wavenumbers, as long-range correlations develop and as bubbles merge. The spectrum also broadens as higher wavenumbers develop through secondary instabilities and nonlinear interactions.

When fluctuations reach the Nyquist wavenumber, they pile up. This occurs in spectral simulations with insufficient numerical dissipation to damp out the highest wave-numbers. The magnitude of the high-wavenumber up-turn provides a measure of aliasing errors. They are inconsequential, provided the up-turn is many orders of magnitude below the peak of the spectrum, as is the case here. The latest time reported for each case very nearly corresponds to the DNS resolution limit (Appendix B).

To assess the evolution of the density spectra in terms of mixing-zone variables, scaled spectra were computed in the $(z=0)$-plane (36), i.e.

$$
\int_{0}^{k_{\mathrm{m}} h / L} E_{\rho}^{*}\left(\frac{k h}{L}\right) \mathrm{d}\left(\frac{k h}{L}\right)=\left\langle\rho^{\prime 2}\right\rangle_{x y} .
$$




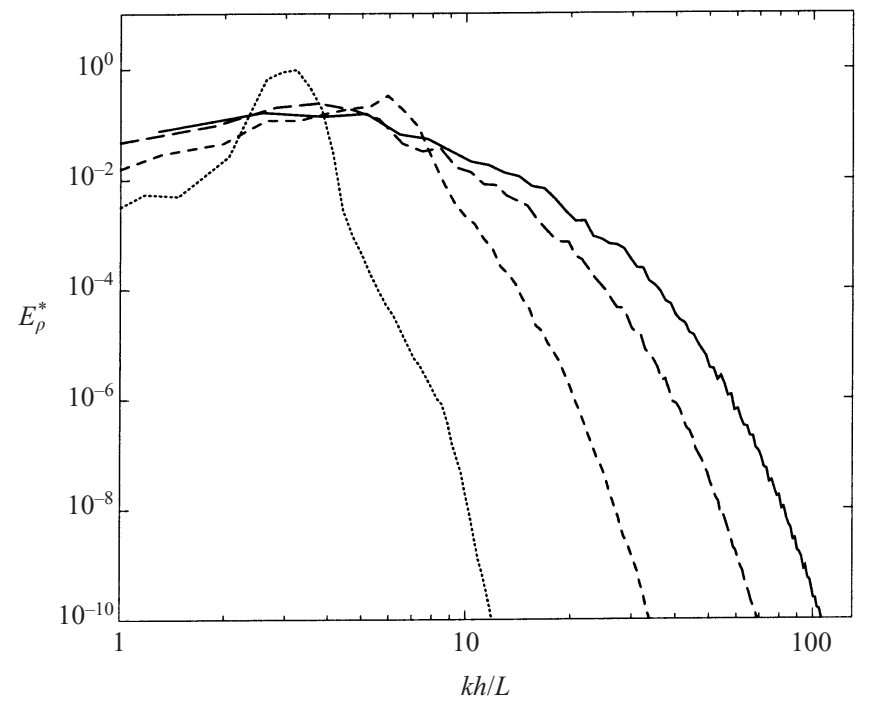

FIGURE 18. Scaled radial spectra, $E_{\rho}^{*}(k h / L)$ in the $(z=0)$-plane for Case C, $v$ s. $k h / L$. Lines of increasing solidity denote increasing time (legend as in figure 17).

The scaled spectra, $E_{\rho}^{*}(k h / L)$, are plotted in figure 18 . The beginning of a collapse at low wavenumbers is evident towards the end of the simulations. The spectra indicate that self-similarity has been attained at low wavenumbers by the end of the simulations and that horizontal large-scale structures scale with mixing-zone height. The full spectra are still evolving, however, consistent with the attainment of only moderate Reynolds numbers in the simulation. It is interesting that differences in nonlinear growth among the three cases persist, despite the fact that the initialperturbation contributions appear completely subsumed in the spectra towards the end of the simulations.

\subsection{Taylor statistics}

Rayleigh-Taylor flow is driven by a directed force field, capable of sustaining anisotropy, at least over a portion of the spectrum. As a consequence, Taylor microscales and Reynolds numbers for this flow must be defined in a manner that accommodates anisotropy. A surrogate microscale in the $i$-direction can be defined in terms of (e.g. Tennekes \& Lumley 1972; Nomura \& Elghobashi 1993)

$$
\lambda_{i}=\left[\frac{\left\langle u_{i}^{2}\right\rangle_{x y}}{\left\langle\left(\partial u_{i} / \partial x_{i}\right)^{2}\right\rangle_{x y}}\right]^{1 / 2} \quad(\text { no sum on } i),
$$

with statistics computed in the $(z=0)$-plane. With statistical isotropy in the $(z=0)$ plane, the $x$ and $y$ microscales are very close and can be averaged to define a single horizontal microscale,

$$
\lambda_{x y}=\frac{\lambda_{x}+\lambda_{y}}{2} .
$$

Figure 19 depicts the temporal growth of the vertical and horizontal Taylor microscales in the $(z=0)$-plane. As can be seen, the initial values for $\lambda / L$ are ranked in order of the dominant- $k$ contribution of the initial perturbation spectra (figure 2).

The flow exhibits strong anisotropy between horizontal and vertical microscales, even in the mixing-zone interior. While horizontal and vertical microscales appear 


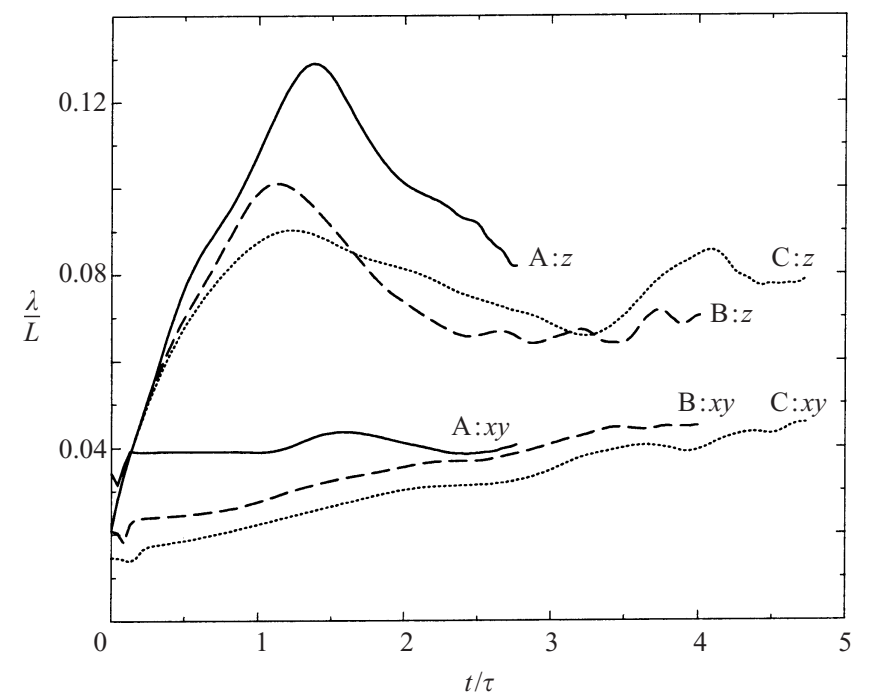

FiguRE 19. Temporal evolution of vertical and horizontal Taylor microscales (38), on the $(z=0)$-plane, for Cases A, B, and C.

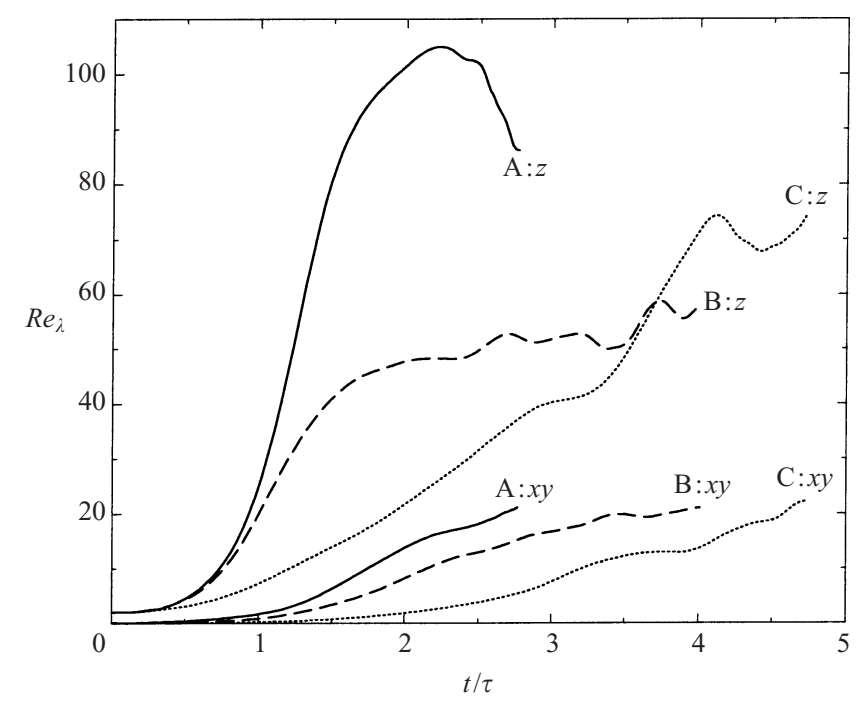

FIGURE 20. Temporal evolution of vertical and horizontal Taylor Reynolds number (39), in the $(z=0)$-plane, for Cases A, B, and C.

to converge towards the end of the simulations, the anisotropic driving term for this flow could sustain anisotropy at the microscale level in the limit $t \rightarrow \infty$.

Figure 20 depicts the temporal evolution of the horizontal and vertical Taylor Reynolds numbers on the $(z=0)$-plane. These are defined as

$$
R e_{\lambda, i}=\frac{\langle\rho\rangle_{x y} \lambda_{i}\left[\left\langle u_{i}^{2}\right\rangle_{x y}\right]^{1 / 2}}{\mu} \quad \text { (no sum on } i \text { ), }
$$

again with spatial averages computed in the $(z=0)$-plane. As with the microscales, horizontal isotropy permits a horizontal Taylor Reynolds number to be defined as 


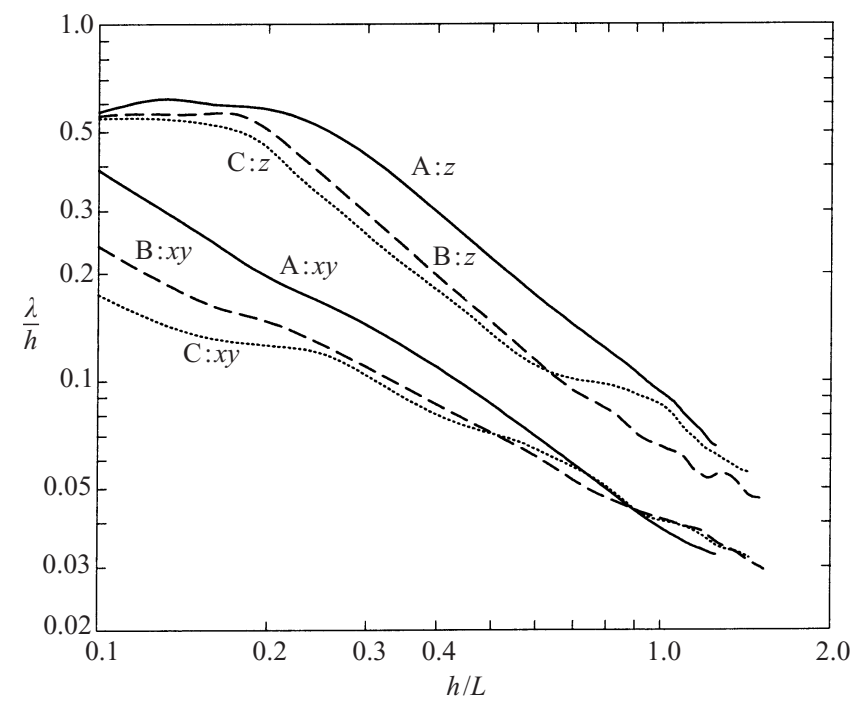

FIGURE 21. Vertical and horizontal Taylor microscales $(38)$, on the $(z=0)$ plane, for Cases A, B, and C.

the average of $R e_{\lambda, x}$ and $R e_{\lambda, y}$, i.e.

$$
\operatorname{Re}_{\lambda, x y}=\frac{R e_{\lambda, x}+R e_{\lambda, y}}{2}
$$

The anisotropy in microscales (figure 19) is also manifest in the Taylor Reynolds numbers, which only partially collapse as a function of time.

Figure 21 depicts the evolution of the Taylor microscales, normalized by mixingzone height, for the three cases, to assess the utility of $h$ as a progress variable. Vertical microscales during the early diffusive-growth stage $(h / L \lesssim 0.2)$ are in accord, when scaled in this fashion, for all three cases. Horizontal microscales, $\lambda_{x y} / h$, agree remarkably well for $h / L \gtrsim 0.6$. The product $h R e_{h}^{-1 / 2}$ provides reasonable late-time ( $h / L \gtrsim 0.6$ ) scaling for the microscales for Case C, for which $\lambda_{z} \approx 3.2 h R e_{h}^{-1 / 2}$, and $\lambda_{x y} \approx 1.7 h R e_{h}^{-1 / 2}$. The late-time prefactors for Case C of 3.2 and 1.7 for the vertical and horizontal microscales, respectively, bracket experimental values of this quantity in other flows, e.g. a prefactor of 2.3 for microscales on the axis of turbulent jets (Dimotakis 2000, equation (19)). The product $h R e_{h}^{-1 / 2}$, however, does not capture the microscale evolution for Cases A and B.

Figure 22 depicts the Taylor Reynolds numbers as a function of $h / L$. As is evident, vertical Taylor Reynolds numbers do not collapse in these coordinates. Conversely, near-perfect collapse is exhibited by the horizontal Taylor Reynolds numbers, over the full range of mixing-zone heights, $h / L$, indicating that interior dynamics responsible for the generation of transverse scales are coupled to (and scaled by) outer scales.

\section{Discussion}

Available RTI linear-stability theory does not capture the early-time (diffusive) evolution discussed above, or the onset and growth-rate ranking in the nonlineargrowth regime. A possible reason for this discrepancy is the diffusive growth of the thin initial composition profiles, independently of the initial perturbations. Diffusion 


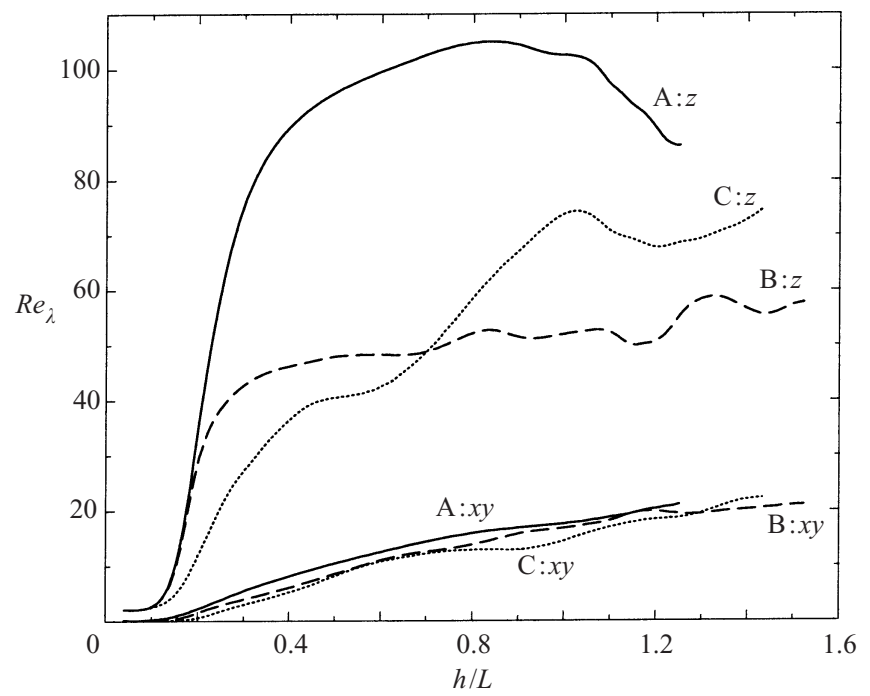

Figure 22. Evolution of vertical and horizontal Taylor Reynolds numbers (39), vs. $h / L$, in the $(z=0)$-plane, for Cases A, B, and C.

initially dominates and alone is able to predict growth of the overall mole-fraction profile thickness, up to times comparable to the outer-scale time, $\tau$, at least for the set of initial perturbations explored in these simulations. RTI between immiscible fluids would not exhibit this early-time diffusive phase and can be expected to behave rather differently.

Near-quadratic (overall) growth is exhibited by the slowest-growing Case C (figure 8), initially seeded by the highest-wavenumber perturbations (figures 1,2 ). Even Case C, however, did not exhibit a quadratic advance of individual (bubble/spike) fronts, cf. (1) and figures 6 and 7, i.e. the advance of the two fronts was not represented particularly well by fixed $\alpha$. The results suggest a dependence on the number of waves in the initial perturbation field (figure 1) and that the initial seeding for Cases A and $B$ may not have permitted sufficient interactions to take place for quadratic growth to develop. Cases A and B, seeded with lower wavenumber perturbations, exhibited faster growth, with overall growth rates (including Case C) in the order of the initial perturbation waves, i.e. $k_{\mathrm{c}} L$. Importantly, all three cases achieved self-similar molefraction profiles towards the end of the simulation (figures 9-11), which, however, were different for each case, indicating that while self-similarity may be necessary for quadratic growth it is not sufficient. At least for the set of initial perturbations considered, these results indicate a sensitive dependence of the nonlinear growth stage on initial conditions, even after allowances are made for the limited statistical confidence in conclusions derived from the small ensemble of runs and interactions between structures within each run. RTI flows possess no inherent length scale, other than those imposed by viscosity and diffusion. Perhaps as a consequence, the imprint of initial perturbation wavenumbers is imposed well into their nonlinear growth regime.

Mixing, which depends on small-scale behaviour, exhibits an even greater sensitivity to initial conditions, varying significantly between cases. Case A, which is seeded by the lowest wavenumber perturbations, exhibits the largest unmixedness, consistent with the notion that entrainment and mixing processes occur at opposite ends of the spatial spectrum. Case $\mathrm{C}$, seeded at the highest wavenumbers, may have attained a 
quasi-asymptotic mixing level, which, however, is different from the values attained by the other two cases.

An investigation of quasi-asymptotic mixing would require investigations extending beyond the mixing transition. With increasing Reynolds number, this transition is expected when outer and inner (viscous) scales become sufficiently separated for a quasi-inviscid range of scales to be accommodated. Experimental data for many flows indicate weaker Reynolds number effects beyond that transition. This requirement translates to a criterion that outer Reynolds numbers must exceed, approximately, $1-2 \times 10^{4}$, or Taylor Reynolds in excess of $100-140$, at a minimum. These are required to generate the high interfacial surface-to-volume ratios needed for good mixing (Dimotakis 1993, 2000). As the outer Reynolds numbers in figures 12 and 13 indicate, such a regime is not attained in the simulations reported here, as also corroborated by the mixing metrics in figures 14 and 16. Nevertheless, mixing and other flow statistics in this simulation appear to have attained values experimentally observed in high Reynolds number flows, such as the chemical-product thickness in gas-phase shear layers and Taylor microscales in turbulent jets, as discussed above.

The coupling between growth and mixing renders RTI mixing-zone evolution potentially dependent on Reynolds number (and Schmidt number). While the maximum Reynolds numbers in these simulations are the highest achieved in DNS of RTI to date they are below the expected mixing-transition values by about a factor of three. Nevertheless, the indications are that the sensitivity of the growth rate to initial conditions will persist to higher Reynolds numbers. Such sensitivity has been reported for turbulent jets, at Reynolds numbers beyond the mixing transition (Miller 1991), as well as high Reynolds number shear layers (Slessor, Bond \& Dimotakis 1998). These flows, and perhaps others, exhibit self-similar but non-universal behaviour in their mixing and other attributes.

Microscale statistics reveal substantial anisotropy, even in the interior of the mixing zone, with vertical microscales roughly twice as large as the horizontal microscales, i.e. $\lambda_{z} / \lambda_{x y} \approx 2$ for $t / \tau \gtrsim 1$. This flow is driven by a directed force field and may exhibit non-isotropic statistics at the microscale level, even at later times.

Each run was executed on 128 CPUs of the ASCI Blue-Pacific (IBM SP2) machine at LLNL. Simulation times, which depend on $\dot{h}$, were, approximately, 200 hours for Case A, 280 hours for Case B, and 300 hours for Case C. The runs required almost 2 months of wall-clock time. The simulations reported here followed an earlier set, which explored various boundary as well as initial conditions, computed at lower resolution and final Reynolds number (Dimotakis et al. 1999). Each simulation required 30 GBytes of RAM. Data dump (restart) files required 2 GBytes of disk storage. 109 files were retained for Case A, 155 for Case B, and 169 for C.

\section{Conclusions}

Three RTI cases were investigated, each with different initial conditions, but otherwise identical in every respect. The runs were initialized with wavenumbers below (Case A), comparable to (Case B), and above (Case C) the most unstable wavenumbers predicted by the linear-stability theory (LST) of Duff et al. (1962). The spectral (wavenumber) content for all three initial perturbations is below the diffusive/viscous cutoff predicted by LST. The simulations attained final outer-scale Reynolds numbers, $R e_{h}=\bar{\rho} h \dot{h} / \bar{\mu}$, ranging from 3000 to 3700 .

Mixing-zone growth exhibits two well-defined regimes: an initial diffusive-growth stage, followed by a nonlinear-growth stage. During the diffusive-growth stage, mixing- 
zone height evolves as, $h(t) \simeq 6.6 \sqrt{\left(t+t_{0}\right) / P e}$, in good agreement with the theoretical prediction corresponding to the $1 \%$ points for the error-function solution of the diffusion equation. The end of the diffusive-growth regime differs substantially, however, among cases. Subsequent growth rates exhibit large differences among the cases, ranked in the same order as the onset of nonlinear growth. In particular, Case A (low- $k$ ) grows the fastest, Case B (mid- $k$ ) is next, with Case C (high-k) the slowest. This is not in the order expected from linear-stability theory. Mixing exhibits an even greater sensitivity to initial conditions, with no obvious scaling nor collapse in any of the three cases. Final Reynolds numbers attained, however, are not high enough to permit inferences about asymptotic-mixing behaviour.

Density-fluctuation spectra computed on the $(z=0)$-plane indicate that the imprint of the initial perturbation spectra is eventually lost. Furthermore, density-fluctuation spectra exhibit good collapse at low wavenumbers in the post-diffusive-growth regime. This is perhaps surprising in view of the persistent influence of the initial conditions on growth and mixing. Taylor microscales and Reynolds numbers also exhibit sensitivity to initial conditions, are non-monotonic in their temporal evolution, and reflect considerable and persistent anisotropy in the flow, at least for the time spanned by these simulations. An important finding is that mixing-zone height, $h$, is a better progress and scaling variable than time. In particular, improved collapse occurs if statistics are compared at the same value of $h$ and if spatial scales are nondimensionalized in terms of $h$.

These results suggest that further numerical and experimental work is necessary to document the behaviour of this flow at higher Reynolds and Schmidt numbers. The apparent sensitivity to initial conditions renders such a quest particularly challenging. Experiments, already difficult to design with controlled initial and boundary conditions, must include a detailed characterization of the initial perturbation environment in which the Rayleigh-Taylor instability develops.

This work was performed under the auspices of the US Department of Energy by the University of California Lawrence Livermore National Laboratory, under contract No. W-7405-Eng-48, the DOE/Caltech ASCI/ASAP subcontract B341492, and the Air Force Office of Scientific Research Grant Nos. F49620-94-1-0353 and F49620 98-1-0052. The authors would like to acknowledge advice from R. D. Henderson on the pressure boundary conditions, discussions and assistance with the text by P. L. Miller and D. Pullin, discussions with D. I. Meiron, and assistance with exploratory computer visualization by S. B. Deusch, S. V. Lombeyda, and J. M. Patton based on the results of the earlier runs.

\section{Appendix A. Poisson solver}

With periodic boundary conditions in $x$ and $y$, the Poisson equation can be Fourier transformed to obtain

$$
\mathscr{F}_{x y}\left\{\frac{\partial^{2} p}{\partial x^{2}}+\frac{\partial^{2} p}{\partial y^{2}}+\frac{\partial^{2} p}{\partial z^{2}}=\Omega(x, y, z)\right\} \Rightarrow-k_{x}^{2} \hat{\hat{p}}-k_{y}^{2} \hat{\hat{p}}+\hat{\hat{p}}^{\prime \prime}=\hat{\hat{\Omega}}\left(k_{x}, k_{y}, z\right),
$$

where $\hat{\hat{p}}^{\prime \prime}=\partial^{2} \hat{\hat{p}} / \partial z^{2}$. Thus $(i$ is the $z$-index of the grid)

$$
\hat{\hat{p}}_{i}^{\prime \prime}=\hat{\hat{\Omega}}_{i}+k^{2} \hat{\hat{p}}_{i}, \quad \text { with } \quad k^{2}=k_{x}^{2}+k_{y}^{2} .
$$

An eighth-order, compact approximation for $\hat{p}_{i}^{\prime \prime}$ can be written as (Collatz 1966; 
Lele 1992),

$$
\begin{aligned}
\beta \hat{\hat{p}}_{i-2}^{\prime \prime}+\alpha \hat{\hat{p}}_{i-1}^{\prime \prime}+\hat{\hat{p}}_{i}^{\prime \prime}+\alpha \hat{\hat{p}}_{i+1}^{\prime \prime}+\beta \hat{\hat{p}}_{i+2}^{\prime \prime} & \\
& =\frac{b}{4 \Delta_{z}^{2}}\left(\hat{\hat{p}}_{i+2}-2 \hat{\hat{p}}_{i}+\hat{\hat{p}}_{i-2}\right)+\frac{a}{\Delta_{z}^{2}}\left(\hat{\hat{p}}_{i+1}-2 \hat{\hat{p}}_{i}+\hat{\hat{p}}_{i-1}\right),
\end{aligned}
$$

where $\Delta_{z}$ is the grid spacing in $z$ and $\alpha=344 / 1179, \beta=23 / 2358, a=320 / 393$, and $b=310 / 393$. Inserting (A 1) into (A 2) and collecting the coefficients of $\hat{\hat{p}}_{i}$ yields the pentadiagonal system,

$$
\begin{aligned}
{\left[\beta k^{2}-\frac{b}{4 \Delta_{z}^{2}}\right] \hat{\hat{p}}_{i-2} } & +\left[\alpha k^{2}-\frac{a}{\Delta_{z}^{2}}\right] \hat{\hat{p}}_{i-1}+\left[k^{2}+\frac{b}{2 \Delta_{z}^{2}}+\frac{2 a}{\Delta_{z}^{2}}\right] \hat{\hat{p}}_{i} \\
& +\left[\alpha k^{2}-\frac{a}{\Delta_{z}^{2}}\right] \hat{\hat{p}}_{i+1}+\left[\beta k^{2}-\frac{b}{4 \Delta_{z}^{2}}\right] \hat{\hat{p}}_{i+2} \\
= & -\left[\beta \hat{\hat{\Omega}}_{i-2}+\alpha \hat{\hat{\Omega}}_{i-1}+\hat{\hat{\Omega}}_{i}+\alpha \hat{\hat{\Omega}}_{i+1}+\beta \hat{\hat{\Omega}}_{i+2}\right] .
\end{aligned}
$$

At points neighbouring boundaries, i.e. $i=2$ and $i=N_{z}-1$, where $N_{z}$ is the number of $z$ grid points, a fourth-order, compact stencil is used in which $\alpha=1 / 10, \beta=0$, $a=6 / 5$ and $b=0$.

The Neumann boundary condition (17), applied at $i=1$ and $i=N_{z}$, is implemented via the second-order approximations:

$$
\begin{gathered}
\frac{3}{2 \Delta_{z}} \hat{\hat{p}}_{1}-\frac{2}{\Delta_{z}} \hat{\hat{p}}_{2}+\frac{1}{2 \Delta_{z}} \hat{\hat{p}}_{3}=-\hat{\hat{p}}_{1}^{\prime}, \\
-\frac{1}{2 \Delta_{z}} \hat{\hat{p}}_{N_{z}-2}+\frac{2}{\Delta_{z}} \hat{\hat{p}}_{N_{z}-1}-\frac{3}{2 \Delta_{z}} \hat{\hat{p}}_{N_{z}}=-\hat{\hat{p}}_{N_{z}}^{\prime},
\end{gathered}
$$

where $\hat{p}_{1}^{\prime}$ and $\hat{\hat{p}}_{N_{z}}^{\prime}$ are the $(x, y)$-transforms of the $z$-derivatives at the walls.

The pentadiagonal matrix for this linear system is well-conditioned, except for $k=0$, in which case it is singular. This situation arises because, with Neumann conditions at both ends of the domain, the solution for the pressure is non-unique since pressure is only defined within a constant. This can be remedied by specifying the mean pressure on either wall, by replacing one of the boundary conditions with $\hat{\hat{p}}_{1}(k=0)=$ constant, or $\hat{\hat{p}}_{N_{z}}(k=0)=$ constant. The value of the constant is inconsequential in incompressible flow as the momentum equation involves only the gradient of $p$.

\section{Appendix B. Resolution considerations}

Spatial derivatives are computed using a Fourier spectral method in $x$ and $y$ and an eighth-order compact scheme in $z$. Because the resolving efficiency of finite-difference schemes is less than that of spectral schemes, the grid spacing in $z$ is reduced relative to the spacing in $x$ and $y$, to compensate for this difference. The proper ratio of grid spacings was determined by the following analysis.

Equations (10), (11), and (12) can be cast in the form

$$
\frac{\partial \phi}{\partial t}+\nabla \cdot \boldsymbol{f}=0
$$

where $\phi=\phi(\boldsymbol{x}, t)$ is a scalar and $\boldsymbol{f}=\boldsymbol{f}(\boldsymbol{x}, t)$ is the associated flux vector. Let $\phi_{i}(t)$ be a spatially discrete sampling of a continuous, band-limited function $\bar{\phi}(\boldsymbol{x}, t)$, i.e. 
$\phi_{i}(t)=\bar{\phi}(i \Delta, t)$, where $i$ is the integer index vector of an infinite, uniform, Cartesian grid with spacing $\Delta$ between nodes. According to the sampling theorem (Shannon $1949)$, if $\bar{\phi}(\boldsymbol{x}, t)$ contains no wavenumbers above the Nyquist frequency, $k_{q}=\pi / \Delta$, the continuous function is completely determined by its samples, i.e.

$$
\bar{\phi}(\boldsymbol{x}, t)=\sum_{i} \phi_{i}(t) \prod_{j=1}^{3} \frac{\sin \left[\pi\left(x_{j}-i_{j} \Delta\right) / \Delta\right]}{\pi\left(x_{j}-i_{j} \Delta\right) / \Delta},
$$

where the sum is over each component of $i$ (a triple sum), and $j$ denotes a particular Cartesian-vector component. Therefore, discrete and continuous band-limited functions contain the same information.

The continuous counterpart of a discrete function can be expressed as a convolution of a raw signal with a filter kernel, subject to certain constraints on the filter (to be derived), i.e.

$$
\bar{\phi}(\boldsymbol{x}, t)=\int_{-\infty}^{\infty} G(|\boldsymbol{x}-\boldsymbol{y}|) \phi(\boldsymbol{y}, t) \mathrm{d}^{3} \boldsymbol{y} \equiv G * \phi .
$$

The governing equation for $\bar{\phi}(\boldsymbol{x}, t)$ is derived by applying (B 3) to (B 1), which yields

$$
\frac{\partial \bar{\phi}}{\partial t}=-G * \nabla \cdot f
$$

Consider now the spatially discrete analogue of (B 1), i.e.

$$
\frac{\partial \phi_{i}}{\partial t}=-\nabla_{\mathrm{d}} \cdot \boldsymbol{f}_{i},
$$

where $\nabla_{\mathrm{d}} \cdot$ denotes a finite-difference approximation to the divergence operator. In order for $\phi_{i}$ and $\bar{\phi}$ to evolve in the same manner, the right-hand sides of (B 4) and (B 5) must be equivalent. Since (B 4) is continuous and (B 5) is discrete, comparison is made by taking continuous and discrete Fourier transforms of (B 4) and (B 5), respectively, both of which yield continuous functions in wavenumber space. Consistency between (B 4) and (B 5) requires

$$
\mathscr{F}\{G * \nabla \cdot f\}=\mathscr{F} \mathrm{d}\left\{\nabla_{\mathrm{d}} \cdot \boldsymbol{f}_{\boldsymbol{i}}\right\},
$$

where $\mathscr{F}\{\cdot\}$ and $\mathscr{F}_{\mathrm{d}}\{\cdot\}$ denote the continuous and discrete Fourier transforms, respectively.

Evaluation of the left-hand side of (B 6) is straightforward. The right-hand side can be evaluated using the modified-wavenumber concept (Vichnevetsky \& Bowles 1982). The result is

$$
\mathrm{i} k_{j} \widehat{f}_{j} \widehat{G}(k)= \begin{cases}\mathrm{i} \tilde{k}_{j} \widehat{f}_{j} & \text { for } k \leqslant k_{q} \\ 0 & \text { for } k>k_{q}\end{cases}
$$

where $\tilde{k}_{j}$ is a modified wavenumber (to be computed for a given finite-difference scheme) and $k=\left(k_{j} k_{j}\right)^{1 / 2}$ is the wavevector magnitude. The directional indices $(j$ subscripts) will now be dropped for notational simplicity. This is allowed since the filtering operation is orthogonal, i.e. the filter can be directionally split and applied sequentially in each direction. From (B 7), the transfer function of the filter must satisfy (Salvetti \& Beux 1998)

$$
\widehat{G}(k)= \begin{cases}\tilde{k} / k & \text { for } k \leqslant k_{q} \\ 0 & \text { for } k>k_{q}\end{cases}
$$

To evaluate $\tilde{k}$ for a given finite-difference scheme, let $l$ and $m$ be integer indices in 


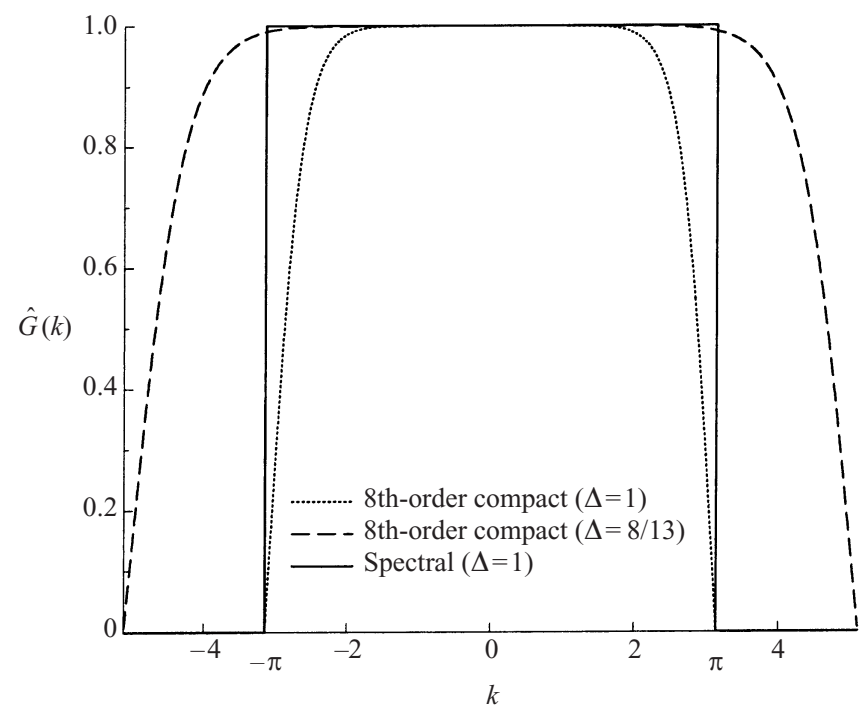

FIGURE 23. Implicit filters of spectral and compact derivatives.

the $j$-direction of the grid. From the Shifting Theorem, the discrete Fourier transform of $f_{l+m}$ is

$$
\widehat{f}_{l+m}=\exp (\mathrm{ikm} \Delta) \widehat{f}_{l} \text {. }
$$

The modified wavenumber for compact finite-difference approximations of the form

$$
\begin{aligned}
\beta f_{l-2}^{\prime}+\alpha f_{l-1}^{\prime}+f_{l}^{\prime} & +\alpha f_{l+1}^{\prime}+\beta f_{l+2}^{\prime} \\
& =\frac{c}{6 \Delta}\left(f_{l+3}-f_{l-3}\right)+\frac{b}{4 \Delta}\left(f_{l+2}-f_{l-2}\right)+\frac{a}{2 \Delta}\left(f_{l+1}-f_{l-1}\right)
\end{aligned}
$$

is obtained by taking the discrete Fourier transform of (B 10) and applying (B 9). The result is

$$
\begin{aligned}
\{1+ & \alpha[\exp (\mathrm{i} k \Delta)+\exp (-\mathrm{i} k \Delta)]+\beta[\exp (\mathrm{i} 2 k \Delta)+\exp (-\mathrm{i} 2 k \Delta)]\} \widehat{f}_{l}^{\prime} \\
=\{ & \frac{c}{6 \Delta}[\exp (\mathrm{i} 3 k \Delta)-\exp (-\mathrm{i} 3 k \Delta)]+\frac{b}{4 \Delta}[\exp (\mathrm{i} 2 k \Delta)-\exp (-\mathrm{i} 2 k \Delta)] \\
& \left.+\frac{a}{2 \Delta}[\exp (\mathrm{i} k \Delta)-\exp (-\mathrm{i} k \Delta)]\right\} \widehat{f}_{l},
\end{aligned}
$$

and hence (Lele 1992)

$$
\tilde{k}=\frac{\widehat{f}_{l}^{\prime}}{\mathrm{i} \widehat{f}_{l}}=\frac{a \sin (k \Delta)+(b / 2) \sin (2 k \Delta)+(c / 3) \sin (3 k \Delta)}{\Delta[1+2 \alpha \cos (k \Delta)+2 \beta \cos (2 k \Delta)]} .
$$

For a simulation to qualify as a DNS, we must have $\bar{\phi}(\boldsymbol{x}, t)=\phi(\boldsymbol{x}, t)$, to a very close approximation. The viscous-stress tensor and the Fickian diffusion term in the governing equations must suffice to damp out all fluctuations outside the band of well-resolved wavenumbers. The maximum Reynolds number that can be achieved in a DNS is directly related to the width of this band of resolved wavenumbers.

The implicit filters for the spectral and eighth-order compact schemes are plotted in figure 23. By decreasing the grid spacing by $8 / 13$, the band of well-resolved wavenumbers for the compact scheme becomes nearly equal to that of the spectral 
scheme. In this fashion, the Nyquist wavenumber is resolved to within $1 \%$, i.e. $\widehat{G}(k=\pi ; \Delta=8 / 13)=0.991$.

\section{REFERENCES}

Andrews, M. J. \& Spalding, D. B. 1990 A simple experiment to investigate two-dimensional mixing by Rayleigh-Taylor instability. Phys. Fluids A 2, 922-927.

Annuchina, N. N., Kucherenko, Yu. A., Neuvazhaev, V. E., Ogibina, V. N., Shibarshov, L. I. \& YakovleV, V. G. 1978 Turbulent mixing at an accelerating interface between liquids of different densities. Izv. Akad. Nauk. SSSR, Mekh Zhidk Gaza 6, 157-160.

Chandrasekhar, S. 1955 The character of the equilibrium of an incompressible heavy viscous fluid of variable density. Proc. Camb. Phil. Soc. 51, 162-178.

Chandrasekhar, S. 1961 Hydrodynamic and Hydromagnetic Stability. Clarendon.

Collatz, L. 1966 The Numerical Treatment of Differential Equations. Springer.

Dalziel, S. B., Linden, P. F. \& Youngs, D. L. 1999 Self-similarity and internal structure of turbulence induced by Rayleigh-Taylor instability. J. Fluid Mech. 399, 1-48.

Dimonte, G. \& Schneider, M. 2000 Density ratio dependence of Rayleigh-Taylor mixing for sustained and impulsive acceleration histories. Phys. Fluids 12, 304-321.

Dimotakis, P. E. 1991 Turbulent free shear layer mixing and combustion. High Speed Flight Propulsion Systems. Progress in Astronautics and Aeronautics, vol. 137, Ch. 5, pp. 265-340.

Dimotakis, P. E. 1993 Some issues on turbulent mixing and turbulence. GALCIT Rep. FM93-1a.

Dimotakis, P. E. 2000 The mixing transition in turbulence. J. Fluid Mech. 409, 69-97.

Dimotakis, P. E., Catrakis, H. J., Cook, A. W. \& Patton, J. M. 1999 On the geometry of twodimensional slices of irregular level sets in turbulent flows. Second Monte-Verita Colloquium on Fundamental Problematic Issues in Turbulence, 22-28 March 1998, Ascona, Switzerland. Published in Trends in Mathematics, pp. 405-418. Birkhäuser.

Duff, R. E., Harlow, F. H. \& Hirt, C. W. 1962 Effects of diffusion on interface instability between gases. Phys. Fluids 5, 417-425.

Durfun, D. R. 1991 The third-order Adams-Bashforth method: an attractive alternative to leapfrog time differencing. Mon. Weath. Rev. 119, 702-720.

Kucherenko, Y. A., Balabin, S. I., Cherret, R. \& HaAs, J. F. 1997 Experimental investigation into internal properties of Rayleigh-Taylor turbulence. Lasers and Particle Beams 15, 25-31.

LELE, S. K. 1992 Compact finite difference schemes with spectral-like resolution. J. Comput. Phys. $103,16-42$.

Linden, P. F., Redondo, J. M. \& Youngs, D. I. 1994 Molecular mixing in Rayleigh-Taylor instability. J. Fluid Mech. 265, 97-124.

Matsuno, T. 1966 Numerical integrations of the primitive equations by a simulated backward difference method. J. Met. Soc. Japan (2) 44, 76-84.

Meshrov, E. E. 1969 Instability of the interface of two gases accelerated by a shock wave. Sov. Fluid Dyn. 4, 101.

Miller, P. L. 1991 Mixing in high Schmidt number turbulent jets. PhD thesis, California Institute of Technology.

Nomura, K. K. \& Elghobashi, S. E. 1993 The structure of inhomogeneous turbulence in variable density nonpremixed flames. Theor. Comput. Fluid Dyn. 5, 153-175.

RAYLEIGH, LORD 1883 Investigation of the character of the equilibrium of an incompressible heavy fluid of variable density. Proc. Lond. Math. Soc. 14, 170-177.

READ, K. I. 1984 Experimental investigation of turbulent mixing by Rayleigh-Taylor instability. Physica D 12, 45-58.

RichtmYer, R. D. 1960 Taylor instability in shock acceleration of compressible fluids. Commun. Pure Appl. Maths 8, 297

Salvetti, M. V. \& BeuX, F. 1998 The effect of the numerical scheme on the subgrid-scale term in large-eddy simulation. Phys. Fluids 10, 3029-3022.

Sandoval, D. L. 1995 The dynamics of variable-density turbulence. PhD thesis, University of Washington.

Shannon, C. E. 1949 Communication in the presence of noise. Proc. IRE 37, 10-21.

Sharp, D. H. 1984 An overview of Rayleigh-Taylor instability. Physica D 12, 3-18. 
Slessor, M. D., Bond, C. L. \& Dimotakis, P. E. 1998 Turbulent shear-layer mixing at high Reynolds numbers: effects of inflow conditions. J. Fluid Mech. 376, 115-138.

TAYLOR, G. I. 1950 The instability of liquid surfaces when accelerated in a direction perpendicular to their planes. Proc. Roy. Soc. Lond. A 201, 192-196.

Tennekes, H. \& Lumley, J. L. 1972 A First Course in Turbulence. MIT Press.

VichneVETSKy, R. \& Bowles, J. B. 1982 Fourier Analysis of Numerical Approximations of Hyperbolic Equations. SIAM.

Young, Y.-N., Tufo, H., Dubey, A. \& Rosner, R. 2001 On the miscible Rayleigh-Taylor instability: two- and three-dimensional. J. Fluid Mech. (in press).

Youngs, D. L. 1984 Numerical simulation of turbulent mixing by Rayleigh-Taylor instability. Physica D 12, 32-44.

Youngs, D. L. 1989 Modelling turbulent mixing by Rayleigh-Taylor instability. Physica D 37, 270-287.

Youngs, D. L. 1994 Numerical simulation of mixing by Rayleigh-Taylor and Richtmyer-Meshkov instabilities. Lasers and Particle Beams 12, 725-750. 